\title{
Hematopoietic mosaic chromosomal alterations and risk for infection among 767,891 individuals without blood cancer
}

Pradeep Natarajan ( $\sim$ pnatarajan@mgh.harvard.edu )

Massachusetts General Hospital

Seyedeh Zekavat

Yale

Shu-Hong Lin

National Cancer Institute

Alexander Bick

Vanderbilt University Medical Center

Aoxing Liu

Institute for Molecular Medicine Finland

Kaavya Paruchuri

Massachusetts General Hospital

Md Mesbah Uddin

Broad Institute

Yixuan Ye

Yale University

Zhaolong Yu

Yale University

Xiaoxi Liu

RIKEN Center for Integrative Medical Sciences

Yoichiro Kamatani

Kyoto University Graduate School of Medicine

James Pirruccello

Massachusetts General Hospital https://orcid.org/0000-0001-6088-4037

Akhil Pampana

Massachusetts General Hospital https://orcid.org/0000-0002-4167-0540

Po-Ru Loh

Brigham and Women's Hospital and Harvard Medical School https://orcid.org/0000-0001-5542-9064

Puja Kohli

Massachusetts General Hospital

Steven McCarroll 
Harvard Medical School https://orcid.org/0000-0002-6954-8184

Benjamin Neale

Massachusetts General Hospital https://orcid.org/0000-0003-1513-6077

\section{Eric Engels}

National Cancer Institute

\section{Derek Brown}

National Cancer Institute

Jordan Smoller

Massachusetts General Hospital

\section{Robert Green}

Harvard Medical School

\section{Elizabeth Karlson}

Brigham and Women's Hospital

\section{Matthew Lebo}

Brigham and Women's Hospital

\section{Patrick Ellinor}

Massachusetts General Hospital https://orcid.org/0000-0002-2067-0533

\section{Scott Weiss}

Brigham and Women's Hospital

\section{Mark Daly}

Institute for Molecular Medicine Finland

\section{Chikashi Terao}

RIKEN https://orcid.org/0000-0002-6452-4095

\section{Hongyu Zhao}

Yale University https://orcid.org/0000-0003-1195-9607

\section{Benjamin Ebert}

Dana-Farber Cancer Institute https://orcid.org/0000-0003-0197-5451

\section{Mitchell Machiela}

National Cancer Institute https://orcid.org/0000-0001-6538-9705

\section{Giulio Genovese}

Broad Institute https://orcid.org/0000-0003-3066-5575

\section{Biological Sciences - Article}

Keywords: Age, DNA genotyping, Aberrant Leukocyte Cell Counts, Diverse Infectious Diseases, Transcriptional Regulatory Sites, Impaired Immunity

Posted Date: November 16th, 2020

DOl: https://doi.org/10.21203/rs.3.rs-100817/v1 
License: (c) (i) This work is licensed under a Creative Commons Attribution 4.0 International License. Read Full License

Version of Record: A version of this preprint was published at Nature Medicine on June 7th, 2021. See the published version at https://doi.org/10.1038/s41591-021-01371-0. 
Hematopoietic mosaic chromosomal alterations and risk for infection among 767,891 individuals without blood cancer

Seyedeh M. Zekavat BS*1-3, Shu-Hong Lin $\mathrm{PhD}^{* 4}$, Alexander G. Bick MD PhD ${ }^{5,2}$, Aoxing Liu $\mathrm{PhD}^{6}$, Kaavya Paruchuri MD ${ }^{2,3,7}$, Md Mesbah Uddin $\mathrm{PhD}^{2,3}$, Yixuan Ye BS ${ }^{1}$, Zhaolong Yu BS ${ }^{1}$, Xiaoxi Liu $\mathrm{PhD}^{8}$, Yoichiro Kamatani $\mathrm{PhD}^{8}$, James P. Pirruccello MD ${ }^{2,3,7}$, Akhil Pampana $\mathrm{MS}^{2,3}$, Po-Ru Loh PhD ${ }^{2,7}$, Puja Kohli MD MMSc ${ }^{10,11}$, Steven A. McCarroll PhD ${ }^{12,13}$, Benjamin Neale $\mathrm{PhD}^{12,14}$, Eric A. Engels MD MPH${ }^{4}$, Derek W. Brown PhD ${ }^{4}$, Jordan W. Smoller MD ScD ${ }^{12,15,16}$, Robert Green MD MPH ${ }^{2,7,9}$, Elizabeth W. Karlson MD MS ${ }^{7,17}$, Matthew Lebo PhD ${ }^{18,19}$, Patrick T. Ellinor MD PhD ${ }^{2,3,7}$, Scott T. Weiss MD MS ${ }^{7,20}$, Mark J. Daly ${ }^{6}$, The Biobank Japan Project, FinnGen Consortium, Chikashi Terao MD PhD ${ }^{8,21,22}$, Hongyu Zhao $\mathrm{PhD}^{1,23}$, Benjamin L. Ebert MD PhD 2,24,25, COVID-19 Host Genetics Initiative, Andrea Ganna PhD ${ }^{6,14,2}$, Mitchell J.

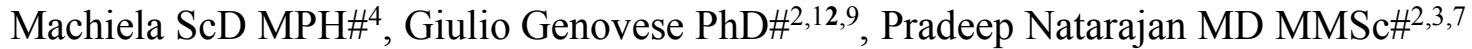

1. Computational Biology \& Bioinformatics Program, Yale University, New Haven, CT

2. Medical and Population Genetics and Cardiovascular Disease Initiative, Broad Institute of Harvard and MIT, Cambridge, MA

3. Cardiovascular Research Center, Massachusetts General Hospital, Boston, MA

4. Division of Cancer Epidemiology and Genetics, National Cancer Institute, Rockville, MD

5. Division of Genetic Medicine, Department of Medicine, Vanderbilt University Medical Center

6. Institute for Molecular Medicine Finland, Helsinki, Finland.

7. Department of Medicine, Brigham and Women's Hospital, Harvard Medical School, Boston, MA

8. Laboratory for Statistical and Translational Genetics, RIKEN Center for Integrative Medical Sciences, RIKEN, Yokohama, Japan

9. Department of Genetics, Department of Medicine, Brigham and Women's Hospital and Harvard Medical School, Boston, MA

10. Division of Pulmonary and Critical Care Medicine, Massachusetts General Hospital, Boston, MA

11. Vertex Pharmaceuticals, Boston, MA

12. Stanley Center, Broad Institute of Harvard and MIT, Cambridge, MA

13. Department of Genetics, Harvard Medical School, Boston, MA

14. Analytic and Translational Genetics Unit, Massachusetts General Hospital, Boston, MA

15. Center for Genomic Medicine, Massachusetts General Hospital, Boston, MA

16. Department of Psychiatry, Harvard Medical School, Boston, MA

17. Division of Rheumatology, Inflammation and Immunity, Brigham and Women's Hospital, Boston, MA

18. Department of Pathology, Brigham and Women's Hospital and Harvard Medical School, Boston, MA

19. Laboratory for Molecular Medicine, Partners Healthcare, Cambridge, MA

20. Channing Division of Network Medicine, Brigham and Women's Hospital, Boston, MA

21. Clinical Research Center, Shizuoka General Hospital, Shizuoka, Japan

22. The Department of Applied Genetics, The School of Pharmaceutical Sciences, University of Shizuoka, Shizuoka, Japan

23. Department of Biostatistics, Yale School of Public Health, New Haven, CT

24. Department of Medical Oncology, Dana-Farber Cancer Institute, Boston, MA, USA

25. Howard Hughes Medical Institute, Boston, MA, USA

* These authors equally contributed to this work. \# These authors equally supervised this work.

Please address correspondence to: Pradeep Natarajan MD, MMSc Massachusetts General Hospital 185 Cambridge Street, CPZN 3.184

Boston, MA 02114

Office: 617-726-1843 


\section{Summary Paragraph:}

3 Age is the dominant risk factor for infectious diseases, but the mechanisms linking the two are

4 incompletely understood ${ }^{1,2}$. Age-related mosaic chromosomal alterations (mCAs) detected from

5 blood-derived DNA genotyping, are structural somatic variants associated with aberrant

6 leukocyte cell counts, hematological malignancy, and mortality ${ }^{3-11}$. Whether mCAs represent

7 independent risk factors for infection is unknown. Here we use genome-wide genotyping of

8 blood DNA to show that mCAs predispose to diverse infectious diseases. We analyzed mCAs

9 from 767,891 individuals without hematological cancer at DNA acquisition across four

10 countries. Expanded mCA (cell fraction $>10 \%$ ) prevalence approached $4 \%$ by 60 years of age

11 and was associated with diverse incident infections, including sepsis, pneumonia, and

12 coronavirus disease 2019 (COVID-19) hospitalization. A genome-wide association study of

13 expanded mCAs identified 63 significant loci. Germline genetic alleles associated with expanded

14 mCAs were enriched at transcriptional regulatory sites for immune cells. Our results link mCAs

15 with impaired immunity and predisposition to infections. Furthermore, these findings may also

16 have important implications for the ongoing COVID-19 pandemic, particularly in prioritizing

17 individual preventive strategies and evaluating immunization responses. 
1 With advancing age comes increased susceptibility to infectious diseases ${ }^{1,2}$. Immunosenescence

2 is the age-related erosion of immune function, particularly with respect to adaptive immunity ${ }^{12-}$

$3 \quad{ }^{15}$. Leukocytes, including T-cells and B-cells, are key mediators of adaptive host defenses against

4 infections, with impaired immune responses increasing risk for infections ${ }^{16-18}$. Age-related

5 mosaic chromosomal alterations (mCAs) detected from blood-derived DNA, are clonal structural

6 somatic alterations (deletions, duplications, or copy neutral loss of heterozygosity) present in a

7 fraction of peripheral leukocytes that can indicate clonal hematopoiesis $(\mathrm{CH})^{3-5} \cdot \mathrm{mCAs}$ are

8 associated with aberrant leukocyte cell counts, and increased risks for hematological malignancy

9 and mortality ${ }^{3-11}$.

11 While the relationship between mCAs and increased hematologic cancer risk is well established ${ }^{3-}$

125 , the impact of mCAs on age-related diminishment in immune function is poorly understood.

13 We hypothesized that mCAs increase risk of infection since mCAs are somatic variants that

14 increase in abundance with age and are associated with alterations in leukocyte count. In this

15 study, we harnessed DNA genotyping array intensity data and long-range chromosomal phase

16 information inferred from 767,891 individuals across four countries to analyze the associations

17 between expanded mCA clones (i.e., mCAs present in at least $10 \%$ of peripheral leukocyte DNA

18 indicative of clonal expansion) and diverse infections, including severe coronavirus disease 2019

19 (COVID-19) from SARS-CoV-2 infection (Figure 1a). To elucidate genetic risk factors for the

20 development of expanded mCA clones, we performed a genome-wide association study (GWAS)

21 in the UK Biobank and subsequent in silico cell-specific, transcriptomic, and pathway analyses. 


\section{Results:}

$3 \quad$ Population characteristics and mCA prevalence

4 A total of 767,891 unrelated, multi-ethnic individuals across the UK Biobank (UKB)

$5 \quad(\mathrm{~N}=444,199)$, Mass General Brigham Biobank (MGBB) $(22,461)$, FinnGen $(\mathrm{N}=175,690)$, and

6 BioBank Japan (BBJ) $(\mathrm{N}=125,541)$ passing genotype and mCA quality control criteria

7 (Supplementary Notes 1-5) were analyzed (Supplementary Table 1). While UKB and BBJ

8 mCA calls were previously performed ${ }^{3,4}$, the MoChA pipeline

9 (https://github.com/freeseek/mocha) was implement to detect mCAs in MGBB and FinnGen

10 (Extended Data Figure 1) from genome-wide genotyping of blood DNA in the present study.

11 Among the UKB participants, mean age at DNA collection was 57 (standard deviation [SD] 8)

12 years, 204,579(46.1\%) were male, 188,875(45.0\%) were prior or current smokers, and 66,551

$13(15.0 \%)$ had a history of solid cancer. In the MGBB, mean age was 55 (SD 17) years, 10,306

$14(45.9 \%)$ were male, 9,094 (40.5\%) were prior or current smokers, and 6,080 (27.1\%) had a

15 history of solid cancer. In FinnGen, mean age was 53 (SD 18) years, 71,000 (40.4\%) were male,

$1642.7 \%$ were prior or current smokers (when smoking status was available), and 31,855 (18.1\%)

17 had a history of solid cancer. In BBJ, mean age was 65 (SD 12) years, 72,186 (57.5\%) were

18 male, and 66,913 (53.3\%) were prior or current smokers, and 25,987 (20.7\%) had a history of

19 solid cancer.

21 In the UKB, among 444,199 unrelated individuals without a known history of hematologic

22 malignancy, 66,011 (14.9\%) carried an mCA (15,350 autosomal) and 12,398 (3.2\%) carried an

23 expanded mCA clone, defined as an mCA mutation present in at least $10 \%$ of peripheral 
1 leukocytes (2,985 autosomal) (Supplementary Table 2). While most of carriers only carried one

2 mCA, 6\% of individuals carried between 2 to 22 non-overlapping mCAs (Supplementary Note

3 6). In the MGBB, across 22,461 unrelated individuals without a history of hematologic cancer,

$4 \quad 3,784(16.8 \%)$ carried an mCA (1,025 autosomal) and 1,026 (5.2\%) carried an expanded mCA

5 clone (337 autosomal). In FinnGen, across 175,690 individuals without a history of hematologic

6 cancer, $22,040(12.5 \%)$ carried an mCA (3,164 autosomal), and 9,558 (5.9\%) carried an

7 expanded mCA clone (1,620 autosomal). In BBJ, across 125,541 individuals without a history of

8 hematologic cancer, only autosomal mCAs were available, with 20,440 carriers (16.3\%) and

$9 \quad 1,676(1.3 \%)$ that carried an expanded clone. (Supplementary Table 2).

11 Consistent with previous reports, the prevalence of mCAs increased with age and was more 12 common among men (Supplementary Note 7,8, and Supplementary Table 3). Across the

13 UKB, MGBB, FinnGen, and BBJ cohorts combined, the prevalence of expanded mCAs was

$14 \quad 0.5 \%$ among individuals $<40$ years, $1.2 \%$ among $40-60$ years, $7.8 \%$ among $60-80$ years, and

$1526.5 \%$ among those greater than 80 years (Figure 1b), the majority of which is due to loss of $\mathrm{X}$

16 in females and loss of Y in males (Supplementary Note 7). The prevalence of expanded

17 autosomal mCAs was $0.27 \%$ among individuals $<40$ years, $0.52 \%$ among $40-60$ years, $1.5 \%$

18 among $60-80$ years, and $4.6 \%$ among those greater than 80 years (Figure 1c).

20 Association of mCAs with hematologic traits

21 We observed a striking association of mCA cell fraction with aberrant cell blood counts acquired

22 at the same visit as blood for genotyping (Figure 1d). Increased mCA cell fraction was

23 associated with overall increased white blood cell count with general consistency across the cell 
1 differential components, with inflections at around cell fraction of 0.1 (Figure 1d). The strongest

2 association across all $\mathrm{mCAs}$ groupings (autosomal/chrX/chrY) with blood counts was between

3 expanded autosomal mCAs and increased lymphocyte count at enrollment (Beta $0.40 \mathrm{SD}$ or 0.25

$4 \times 10^{9}$ cells/L; $95 \%$ CI 0.36 to 0.44 SD; $\mathrm{P}=4.2 \times 10^{-84}$ ) (Extended Data Figure 2, Supplementary

5 Note 9).

6

7 Similarly, incident hematologic cancer risk was also strongly dependent on cell fraction

8 (Extended Data Figure 3a,b). We reproduced the associations of mCAs with hematologic

9 cancers with similar effects as previously described in the $\mathrm{UKB}^{4,5}$. We found that expanded

10 autosomal mCAs with cell fraction $>10 \%$ were most strongly associated with incident

11 hematologic cancer (Extended Data Figure 3c), with the strongest association being for

12 incident chronic lymphocytic leukemia (HR $121.9 ; 95 \%$ CI 93.6 to $158.9 ; \mathrm{P}=4.2 \times 10^{-277}$ );

13 although an association with myeloid leukemia was also present (HR 12.3; 95\% CI 7.7 to 19.7;

$14 \mathrm{P}=2.3 \times 10^{-25}$ ) (Supplementary Figure 11). While expanded chrX and chrY mCAs were also

15 associated with chronic lymphocytic leukemia, their effects were considerably lower (chrX: HR

$1624.1,95 \%$ CI 5.8 to $99.9, \mathrm{P}=1.1 \times 10^{-5}$ and chrY: HR 2.0, 95\% CI 1.0 to $4.0, \mathrm{P}=0.038$ ) (Extended

17 Data Figure 3c).

19 Associations with diverse infections

20 mCA presence across the genome was associated with diverse incident infections (defined in

21 Supplementary Data 1,2) (HR 1.06; 95\% CI 1.04 to 1.09; $\left.\mathrm{P}=8.6 \times 10^{-8}\right)($ Supplementary Note

22 10), independent of age, age ${ }^{2}$, sex, smoking status, and first 10 principal components of ancestry

23 in the combined UKB, MGBB, and FinnGen meta-analysis. The dependence of this association 
1 with mCA cell fraction is further visualized in Figure 2a,b, which shows an increase in

2 proportion of incident infection cases and incident sepsis cases with cell fraction, with greater

3 slopes observed at approximately cell fraction $>10 \%$. Accordingly, the associations across

4 diverse infections were stronger for expanded mCA clones, (HR 1.12; 95\% CI 1.1 to 1.2;

$\left.5 \mathrm{P}=6.3 \times 10^{-7}\right)$ (Figure 2c). Furthermore, among expanded mCA clones, the strongest association

6 was observed among expanded autosomal mCAs (HR 1.3; 95\% CI 1.1 to $1.4 ; \mathrm{P}=1.8 \times 10^{-7}$ )

7 (Figure 2c). Accounting for multiple hypothesis testing, expanded autosomal mCAs were

8 significantly associated with sepsis (HR $2.7 ; 95 \%$ CI 2.3 to $3.2 ; \mathrm{P}=3.1 \times 10^{-28}$ ), respiratory system

9 infections (HR $1.4 ; 95 \%$ CI 1.2 to $1.5 ; \mathrm{P}=3.8 \times 10^{-10}$ ), digestive system infections (HR $1.5 ; 95 \%$

10 CI 1.3 to $1.7 ; \mathrm{P}=2.2 \times 10^{-9}$ ), and genitourinary system infections (HR $1.3 ; 95 \%$ CI 1.1 to 1.4 ;

$\left.11 \mathrm{P}=3.7 \times 10^{-4}\right)$ (Figure 2c). The specific expanded autosomal mCAs implicated for infection were

12 diverse in nature - across all chromosomes, of different sizes, and mixed across gain, loss, and

13 copy-number neutral loss of heterozygosity (CNN-LOH) mCAs (Extended Data Figure 4).

14 Further associations across 20 specific infectious disease subcategories are enumerated in

15 Supplementary Note 11. For sex chromosome mCAs, none of the incident infections achieved

16 statistical significance $(\mathrm{P}<0.005)$ in meta-analysis across the three cohorts; however, respiratory

17 infections were suggestively associated (expanded chrX: HR $1.5 ; 95 \%$ CI 1.01 to $1.9 ; \mathrm{P}=0.0068$;

18 expanded chrY: HR 1.09; 95\% CI 1.0 to 1.2; $\mathrm{P}=0.005$ ) (Supplementary Figure 12).

21 Risks for incident fatal infections were assessed in BBJ since non-fatal incident infectious

22 disease events are currently unavailable in BBJ. Among individuals without any cancer history in

23 BBJ, autosomal mCAs showed nominal associations with fatal incident infections (HR 1.12, 
$195 \% \mathrm{CI} 1.0$ to $1.2 \mathrm{P}=0.04$ ), with expanded autosomal mCAs being associated with incident

2 sepsis mortality (HR 2.0; 95\% CI 1.0 to 4.2; $\mathrm{P}=0.05$ ) (Supplementary Table 4, Extended Data

3 Figure 5), as well as pneumonia history (OR 1.3; 95\% CI: 1.1 to 1.5 ; $\mathrm{P}=0.0019$ ).

4

5 Sensitivity analysis for the association of expanded autosomal mCAs and incident sepsis found

6 that the association was consistently significant across different age groups (Supplementary

7 Note 13), and that it was additionally independent of a 25 -factor smoking covariate ${ }^{10}$, body mass

8 index, type 2 diabetes mellitus, leukocyte count, lymphocyte count, and lymphocyte percentage

9 (Supplementary Table 5).

11 Stratified analyses indicated expanded autosomal mCAs in individuals with cancer prior to

12 infection (either any solid tumors, or hematologic malignancy after time of blood draw for

13 genotyping) conferred stronger effects for sepsis (HR 2.8; 95\% CI 2.3 to $3.4 ; \mathrm{P}=9.7 \times 10^{-26}$ ) and

14 respiratory system infections (HR $1.6 ; 95 \%$ CI 1.4 to $1.8 ; \mathrm{P}=6.1 \times 10^{-12}$ ) compared to individuals

15 without a prior cancer history (sepsis: $\mathrm{HR} 1.3 ; 95 \%$ CI 0.8 to $2.0 ; \mathrm{P}=0.33$, $\mathrm{P}_{\text {heterogeneity }}=0.001$;

16 respiratory system infections: HR $1.2 ; 95 \%$ CI 1.0 to $\left.1.3 ; \mathrm{P}=0.045, \mathrm{P}_{\text {interaction }}=0.001\right)$ (Extended

17 Data Figure 6,7; Supplementary Note 14,15). This interaction was driven by prevalent solid

18 cancer, not hematologic cancer after DNA acquisition for mCA genotyping (Supplementary

19 Table 6). Further multivariable adjustment indicated that incident sepsis and infection were

20 independent of chemotherapy, neutropenia, aplastic anemia, decreased white blood cell count,

21 bone marrow or stem cell transplant, and radiation effects prior to infection (with these

22 phenotypes defined using ICD-10 and ICD-9 phecode groupings ${ }^{19}$ ) (Extended Table 1). 
2 Across 719 COVID-19 hospitalized cases in the UKB, 44 individuals (6\%) carried an expanded 3 mCA clone at time of enrollment (in 2010), versus 3\% among 337,877 controls. Adjusting for 4 age, age ${ }^{2}$, sex, prior or current smoking status, and principal components of ancestry, expanded 5 mCAs were associated with COVID-19 hospitalizations (OR 1.6; 95\% CI 1.1 to $2.2 ; \mathrm{P}=0.0082$ ),

6 with similar effects with expanded autosomal mCAs (OR 2.2; 95\% CI 1.2 to $4.1 ; \mathrm{P}=0.02$ )

7 (Figure 3a). Analyses in FinnGen showed evidence of independent replication. The meta-

8 analyzed associations across UKB and FinnGen of expanded autosomal mCAs on COVID-19

9 hospitalization was OR 2.4, 95\% CI 1.3 to 4.5, $\mathrm{P}=0.004$ (Figure 3a,b). Similar to prior

10 phenotypes, the fraction of individuals hospitalized with COVID-19 increased with cell fraction, 11 with particularly strong slopes after cell fraction $>10 \%$ (Figure 3c). In the UKB, further

12 sensitivity analysis was performed; the associations persisted with additional adjustment for

13 normalized Townsend deprivation index, normalized body mass index, type 2 diabetes mellitus,

14 hypertension, coronary artery disease, any cancer, asthma, and chronic obstructive pulmonary

15 disease (Extended Data Figure 8a). Additionally, similar associations were observed in the

16 UKB when comparing COVID-19 hospitalization to tested negative controls, COVID-19

17 positive to all from English provinces and, COVID-19 positive to tested negative controls

18 (Extended Data Figure 8b). Similar to the diverse nature of mCA clones observed in cases of 19 incident infection, specific mCA clones carried by COVID-19 hospitalized individuals were also

20 diverse in nature - across multiple chromosomes, a wide range of sizes, and both gain, loss, and

21 CNN-LOH copy changes (Figure 3d). Similar effects associations effects of expanded mCAs

22 with COVID-19 across expanded mCAs were also observed with incident pneumonia in the

23 UKB (Extended Data Figure 8c). 
2 Germline genetic predisposition to expanded mCAs

3 To further elucidate causal factors for expanded mCA clones, we performed a genome-wide

4 association study (GWAS) in the UKB. We identified 63 independent genome-wide significant

5 loci $\left(\mathrm{r}^{2}<0.1\right.$ across $1 \mathrm{MB}$ windows of the genome) (Figure 4a, Supplementary Data 3). Indeed,

6 across the 63 germline variants, significant correlation was seen between different mCA

7 categories (Supplementary Note 16), suggesting the presence of shared germline genetic

8 variants predisposing to mCAs across the genome. Follow-up analyses using an additive

9 polygenic risk score comprised of 156 independent genome-wide significant variants associated

10 with mosaic loss-of-chromosome $\mathrm{Y}$ (mLOY) from males from a prior study in the $\mathrm{UKB}^{20}$, found

11 significant associations with expanded autosomal mCAs and expanded $\mathrm{ChrX} \mathrm{mCAs} \mathrm{in} \mathrm{females,}$

12 further highlighting the shared germline contributors towards mCAs across the genome

13 (Supplementary Note 17). TWAS combining the expanded mCA GWAS results with

14 GTEXv8 ${ }^{21}$ whole blood expression quantitative trait loci (eQTLs) using UTMOST $^{22}$ prioritized

1562 genes $\left(\mathrm{P}<3.2 \times 10^{-6}\right)$ promoting expanded mCA development (Figure $\left.4 \mathbf{b}\right)$. While gene

16 enrichment analyses with the Elsevier Pathway Collection did not identify significantly

17 associated pathways after multiple testing correction, top pathways were linked to DNA damage

18 repair and lymphoid processes (Extended Data Figure 9a). The corresponding GWAS locus-

19 zoom plots for some of these immune-related genes are shown in Extended Data Figure 9b. To

20 prioritize tissues most implicated by these loci, tissue enrichment analyses using GenoSkyline-

21 Plus were performed. Significant enrichment was identified in immune-specific epigenetic and

22 transcriptomic functional regions of the genome $\left(\mathrm{P}=7.1 \times 10^{-9}\right)$ (Figure 4c). Further stratification 
1 of the immune category identified specific enrichment for CD4+ T-cells $(\mathrm{P}=0.00098)$ (Figure 2 4d). 


\section{Discussion:}

3 Across four geographically distinct biobanks comprising 767,891 individuals without known

4 hematologic malignancy, clonal hematopoiesis $(\mathrm{CH})$ represented by expanded mCAs is

5 increasingly prevalent with age but not readily detectable by conventional medical blood tests. In

6 addition to strongly predicting future risk of hematologic malignancy, expanded mCAs were also

7 associated with risk for diverse incident infections, particularly sepsis and respiratory infections.

8 These findings were robust across age, sex, tobacco smoking, and were strongest among those

9 who develop cancer. Consistent with these observations, expanded mCAs were also associated

10 with increased odds for COVID-19 hospitalization.

12 These results support several conclusions. First, mCA-driven $\mathrm{CH}$ is a potential risk factor for

13 infection. Recent work showed that $\mathrm{CH}$ with myeloid malignancy driver mutations, also referred

14 to as 'clonal hematopoiesis of indeterminate potential' (CHIP), predisposes to myeloid

15 malignancy and coronary artery disease ${ }^{23-27}$. Meanwhile, $\mathrm{CH}$ with larger chromosomal

16 alterations (i.e., mCAs) predisposes primarily to lymphoid malignancy but not coronary artery

17 disease $\mathrm{e}^{3-5,8,9}$. Our observations suggest $\mathrm{CH}$ defined by the presence of mCAs is a risk factor for

18 infection. Since the relationship between mCAs and infection risk was not substantially

19 attenuated when adjusting for leukocyte or lymphocyte counts at baseline visit, the impact of

20 mCAs on infection risk likely acts through mechanisms independent of the impact of $\mathrm{CH}$ on cell

21 counts. For example, as mCAs alter gene dosage (e.g., via duplications and deletions) and

22 remove allelic heterogeneity (e.g., copy neutral loss-of-heterozygosity events) in leukocytes,

23 potential impacts on the differentiation, function, and survival of leukocytes are mechanisms that 
1 could lead to altered infection risk. Our germline analyses specifically implicate lymphoid

2 tissues. In particular, many of the mCA susceptibility loci are the same as those found in chronic

3 lymphocytic leukemia, a condition in which lymphocyte differentiation and function is altered

4 promoting infection risk $^{28-31}$. Therefore, molecular changes in leukocytes that promote clonal

5 expansion may occur at the expense of reduced ability to combat infection.

7 Second, the infectious disease risk associated with mCAs is exacerbated in the setting of cancer.

8 It is well-established that mCAs in blood-derived DNA increase risk for hematologic cancer $^{3-5}$.

9 Furthermore, recent evidence suggests an association between mCAs detected in blood-derived

10 DNA and increased risk of select solid tumor ${ }^{7,10,32}$. Our analysis identified an interaction between

$11 \mathrm{mCAs}$ and prior cancer diagnosis that amplified sepsis and pneumonia risk. Importantly, this

12 interaction was restricted to individuals with solid cancers, not antecedent blood cancer. While

13 this observation could be partially due to synergistic immunosuppressive side effects of cancer

14 therapies $^{33}$, the observed associations persisted despite adjustment for many of these treatments.

15 Alternatively, abnormal regulation of immune inflammatory pathways that release cytokines and

16 inflammatory cells may create chronic states of inflammation in individuals with mCAs ${ }^{34,35}$.

17 Surveillance for expanded mCA clones, particularly among those who develop solid cancer, may

18 help identify individuals at high risk for infection that could benefit from targeted interventions.

20 Third, our findings could have particular relevance for the ongoing COVID-19 pandemic. We

21 observed that mCAs are associated with elevated risk for COVID-19 hospitalization, with greater

22 than two-fold risk linked to expanded autosomal mCAs. Maladaptive immune responses,

23 particularly in leukocytes, increase risk for severe COVID-19 infections ${ }^{36-39}$. Awareness of 
1 COVID-19 risk associated with mCAs may help with the prioritization of emerging prophylactic

2 treatments and initial vaccination programs. However, whether immune response to conventional

3 vaccination approaches is altered in the context of mCAs deserves further study.

4

5 This analysis of mCAs and infection had some limitations. First, our study only measures mCAs

6 at one time point for each participant. While our sampled mCA time point is likely correlated

7 with $\mathrm{CH}$ at time of infection, $\mathrm{CH}$ dynamically changes over time potentially leading to

8 differences in cellular fraction or additional undetected events that were acquired prior to

9 infection. Second, we cannot rule out the possibility of undiagnosed hematologic malignancy

10 among individuals with mCAs with only blood DNA. However, given the observed prevalence

11 of mCAs ( $4 \%$ by age 60 years) among individuals without diagnosed hematologic malignancy

12 and general scarcity of hematologic malignancy in the general population, we anticipate

13 undiagnosed hematologic malignancy at DNA acquisition to be uncommon. Third, despite the

14 robust adjustment and sensitivity analyses performed in our statistical analysis, including

15 adjustment for chemotherapy, bone marrow transplant, radiation, and other features associated

16 with poor cancer prognosis (neutropenia, aplastic anemia, decreased white blood cell count), we

17 cannot completely rule out the impact of residual confounding in our results from unknown or

18 unmeasured sources. Consistency across cohorts and infection types and biologic plausibility

19 mitigates this possibility, but functional studies testing the hypothesis that these represent causal

20 relationships merit consideration.

22 In conclusion, we report evidence for increased susceptibility to a spectrum of infectious diseases

23 in individuals carrying mCAs in a detectable fraction of leukocytes. The impacts of mCA on 
1 infection risk are systemic, with increased susceptibility to infection observed for a variety of 2 organ systems, including severe COVID-19 presentations. 


\section{Online Methods:}

\section{Study samples}

4 A total of 767,891 individuals across four biobanks were analyzed: UK Biobank (UKB), Mass

5 General Brigham Biobank (MGBB), FinnGen, and Biobank Japan (BBJ) ${ }^{40-42}$. Across all three

6 cohorts, written informed consent was previously obtained from all participants. Individuals with

7 known hematologic cancer at time of or prior to blood draw for genotyping were removed from 8 all analyses. Additional information on each cohort is provided in Supplementary Note 1.

Mosaic chromosomal alteration detection

11 Mosaic chromosomal alteration (mCA) detection has been previously described in the $\mathrm{UKB}^{4,5}$

12 and $\mathrm{BBJ}^{3}$. mCA detection in the MGBB and FinnGen were performed with the Mosaic

13 Chromosomal Alterations (MoChA) software and pipeline (https://github.com/freeseek/mocha).

14 Briefly, genotype intensities were transformed to $\log _{2}$ (R ratio) (LRR) and B-allele frequency

15 (BAF) values to estimate total and relative allelic intensities, respectively, as previously

16 described ${ }^{43}$. Further details regarding the mCA detection are provided in Supplementary Note

17 1-5. Across all three studies, expanded mCA refers to the presence of at least one detectable

18 mCA present in $>10 \%$ of circulating leukocytes (e.g., cell fraction $>10 \%$ ). A $10 \%$ cell fraction

19 threshold was employed since this has been previously linked to greater clonal haematopoiesis-

20 related risk for incident mortality ${ }^{10}$ and myocardial infarction ${ }^{23}$, additionally this subset was

21 observed to most strongly associate with phenotypes in the UK Biobank including aberrant blood

22 cell counts, incident hematologic cancer, and incident infections (Figure 1d, 2a,b, Extended

23 Data Figure 3ab). Autosomes and sex chromosomes were also separately considered; only

24 autosomal mCAs were available for BBJ. 
2 Clinical outcomes

3 Definitions for infection outcomes are detailed in Supplementary Data 1,2. In the UKB, the

4 first reported occurrences over median 8-year follow-up in Category 2410 were used as

5 categorized by the UKB which maps primary care data, ICD-9 and ICD-10 codes from hospital

6 inpatient data, ICD-10 codes in death register records, and self-reported medical conditions

7 reported at the baseline, to ICD-10 codes. For each set of phenotypes grouped by organ system

8 or by category, the time to first incident event after baseline examination in individuals free of

9 prevalent history of each disease category was used. In the MGBB, electronic health record data

10 was used to define incident ICD-10 codes grouped in the same fashion after DNA collection date

11 over a median 3-year follow-up. In FinnGen, phenotypes were grouped together across ICD-8,

12 ICD-9, and ICD-10 codes (Supplementary Data 2), with incident infections defined after DNA

13 collection date over a median 3-year follow-up. In BBJ, analyses were performed using fatal

14 incident events attributed to diverse infection outcomes in Supplementary Data 1 since non-

15 fatal incident events were not available; additionally, analyses for pneumonia were performed

16 using history of pneumonia prior to genotyping, based on interviews and medical record

17 reviews ${ }^{41}$. Other clinical phenotypes defined in the UKB, MGBB, and FinnGen are detailed in

18 Supplementary Note 1 and Supplementary Data 6-8.

20 UKB coronavirus disease 2019 (COVID-19), from SARS-CoV-2 infection, phenotypes used in

21 the present analysis were downloaded on July 27, 2020. SARS-CoV-2 infection was determined

22 by polymerase chain reaction from nasopharygeal, oropharyngeal, or lower respiratory samples

23 obtained between March 16, 2020 and July 17, 2020. COVID-19 hospitalized cases were defined 
1 as any individual with at least one positive test who also had evidence for inpatient

2 hospitalization (Field 40100). Controls included two sets: (1) participants from UKB English

3 recruitment centers who were not known to have COVID-19, which were individuals with

4 negative or no known SARS-CoV-2 testing or (2) participants with a negative SARS-CoV-2 test.

5 Individuals with COVID-19 of unknown or low severity (i.e., at least one positive SARS-CoV-2

6 test without a known hospitalization) were excluded from the primary analyses.

7 Replication was performed in FinnGen where SARS-CoV-2 infection was determined either by

8 polymerase chain reaction or by antibodies for samples obtained between March 2, 2020 and

9 July 27, 2020. Across both cohorts, individuals who died prior to March 1, 2020, and therefore

10 were not at risk for COVID-19 infection, were excluded from COVID-19 analyses.

\section{Statistical methods for infection associations}

13 Association analyses of expanded mCAs with primary incident infection across 10 main

14 infectious disease organ system categories (listed under "organ system" in Supplementary Data

15 1) were performed using Cox proportional hazards models, adjusting for age, age ${ }^{2}$, sex, ever

16 smoking status, and principal components 1-10 from the genotyping data. Time since DNA

17 collection was used as the underlying timescale. The proportional hazards assumption was

18 assessed by Schoenfeld residuals and was not rejected. Individuals with a history of

19 hematological cancer prior to DNA collection were excluded. P-value threshold for significance

20 among the primary organ system infection analyses was two-sided $0.05 / 10=0.005$ to account for

21 multiple hypothesis-testing. Secondary and sensitivity analyses are detailed in the

22 Supplementary Note 1. Analyses of incident events were performed separately in each biobank

23 using the survival package in R (version 3.5, R Foundation, Vienna, Austria). Meta-analyses of 
1 the UKB, MGBB, and FinnGen results were performed using a fixed effects model from the

2 meta package.

4 For UKB COVID-19 analyses, logistic regression was performed to estimate the association

5 between expanded mCAs and COVID-19 hospitalization using the aforementioned phenotype

6 definition, adjusting for sex, age, age ${ }^{2}$, smoking status, and the first ten principal components

7 from the genotyping data. As above, individuals with prevalent hematologic cancer were

8 excluded from analyses. For the COVID-19 analyses, statistical significance was assigned at

9 two-sided p-value $<0.05$. Secondary multi-variable models were additionally adjusted for

10 normalized Townsend deprivation index ${ }^{44}$, inverse rank normalized body mass index at baseline,

11 type 2 diabetes mellitus, hypertension, coronary artery disease, any cancer, asthma, and chronic

12 obstructive pulmonary disease.

\section{Genome-wide association study}

14 GWAS was performed using Hail-0.2 software (https://hail.is/) on the Google cloud. Variants

15 were filtered to high-quality imputed variants (INFO score $>0.4$ ), with minor allele frequency

$16>0.005$, and with Hardy-Weinberg Equilibrium $\mathrm{P} \geq 1 \times 10^{-10}$, as previously performed. A Wald-

17 logistic regression model was used for analysis, adjusting for age, age ${ }^{2}$, sex, ever smoking, PC1-

1810 , and genotyping array. Significant, independent loci were identified using $\mathrm{P}<5 \times 10^{-8}$ and

19 clumping in Plink-2.0 using an $\mathrm{r}^{2}$ threshold of 0.1 across $1 \mathrm{MB}$ genomic windows using the 1000-

20 Genomes Project European reference panel. An additive mLOY polygenic risk score was

21 developed as such: $\sum_{i=1}^{63}$ Beta $\times S N P_{i j}$, where Beta is the weight for each of the 156

22 independent genome-wide significant variants previously identified in UKB males ${ }^{20}$ and $S N P_{i j}$ is

23 the number of alleles (i.e., 0,1 , or 2) for $S N P_{i}$ in female $j$ in the UKB. 


\section{Cell-type enrichment analyses}

2 We applied partitioned LD score regression using the LDSC software ${ }^{45}$ to perform enrichment

3 analysis using the expanded mCA GWAS summary statistics in combination with tissue-specific

4 epigenetic and transcriptomic functionality annotations from GenoSkyline-Plus ${ }^{22}$. In addition to

5 the baseline annotations for diverse genomic features as suggested in the LDSC user manual, we

6 specifically examined the enrichment signals on two tiers of annotations of different resolutions:

7 GenoSkyline-Plus functionality scores of 7 broad tissue clusters (immune, brain, cardiovascular,

8 muscle, gastrointestinal tract, epithelial, and others); and GenoSkyline-Plus functionality scores

9 of 11 tissue and cell types within the immune cluster (listed in Figure 8D).

10 Transcriptome-wide association and pathway enrichment analysis

11 Transcriptome-wide association was performed using the expanded mCA GWAS summary

12 statistics in combination with the UTMOST ${ }^{46}$ whole blood model updated to GTEXv8 (N=670).

13 Significant genes were identified using a Bonferroni cutoff of $\mathrm{P}<0.05 / 15,625$ or $3.2 \times 10^{-6}$.

14 Pathway enrichment analyses was performed using genes with TWAS $\mathrm{P}<0.001$ using the

15 Elsevier Pathways through the EnrichR web server ${ }^{47}$.

16

17

18 


\section{Acknowledgements:}

3 Thanks to Chris Whelan, Chris Llanwarne, Jason Cerrato, Kyle Vernest, and Khalid Shakir and

4 many other members of the Terra/Cromwell team for their help and advice in the development of

5 the MoChA pipeline. Thanks to Petr Danecek for implementing critical features needed in

6 BCFtools. Thanks to Stephen Chanock for critical input and comments. Thanks to Erikka

7 Loftfield for assistance with the 25-level smoking adjustment variable. Thanks to the participants

8 and staff of the UKB, MGBB, and BBJ. UKB analyses were conducted using Applications 7089

9 and 21552.

\section{Funding:}

13 P.N. is supported by a Hassenfeld Scholar Award from the Massachusetts General Hospital, and 14 grants from the National Heart, Lung, and Blood Institute (R01HL1427, R01HL148565, and

15 R01HL148050). P.N. and B.L.E. are supported by a grant from Fondation Leducq (TNE-

16 18CVD04). S.M.Z is supported by the NIH National Heart, Lung, and Blood Institute

17 (1F30HL149180-01) and the NIH Medical Scientist Training Program Training Grant

18 (T32GM136651). A.G.B. is supported by a Burroughs Wellcome Fund Career Award for

19 Medical Scientists. G.G is supported by NIH grant R01 HG006855, NIH grant R01 MH104964,

20 and the Stanley Center for Psychiatric Research. J.P.P is supported by a John S LaDue Memorial

21 Fellowship. K.P. is supported by NIH grant 5-T32HL007208-43. P.T.E. is supported by

22 supported grants from the National Institutes of Health (1RO1HL092577, R01HL128914,

23 K24HL105780), the American Heart Association (18SFRN34110082), and by the Foundation 
1 Leducq (14CVD01). P.-R.L. is supported by NIH grant DP2 ES030554 and a Burroughs

2 Wellcome Fund Career Award at the Scientific Interfaces. This work was supported by the

3 Intramural Research Program of the National Cancer Institute, National Institutes of Health,

4 extramural grants from the National Heart, Lung, and Blood Institute, and Fondation Leducq.

5 The opinions expressed by the authors are their own and this material should not be interpreted

6 as representing the official viewpoint of the U.S. Department of Health and Human Services, the

7 National Institutes of Health, or the National Cancer Institute.

8 Competing Interests:

9 P.N. reported grants from Amgen during the conduct of the study and grants from Boston

10 Scientific; grants and personal fees from Apple; personal fees from Novartis and Blackstone Life

11 Sciences; and other support from Vertex outside the submitted work. P.T.E. has received grant

12 support from Bayer AG and has served on advisory boards or consulted for Bayer AG, Quest

13 Diagnostics, MyoKardia and Novartis, outside of the present work. S.M.Z., S-H.L., M.J.M.,

14 G.G., and P.N. have filed a patent application (serial no. 63/079,74) on the prediction of infection

15 from mCAs. G.G. and S.A.M. have filed a patent application (PCT/WO2019/079493) for the

16 MoChA mCA detection method employed in the present study. No other disclosures were

17 reported. 
a.

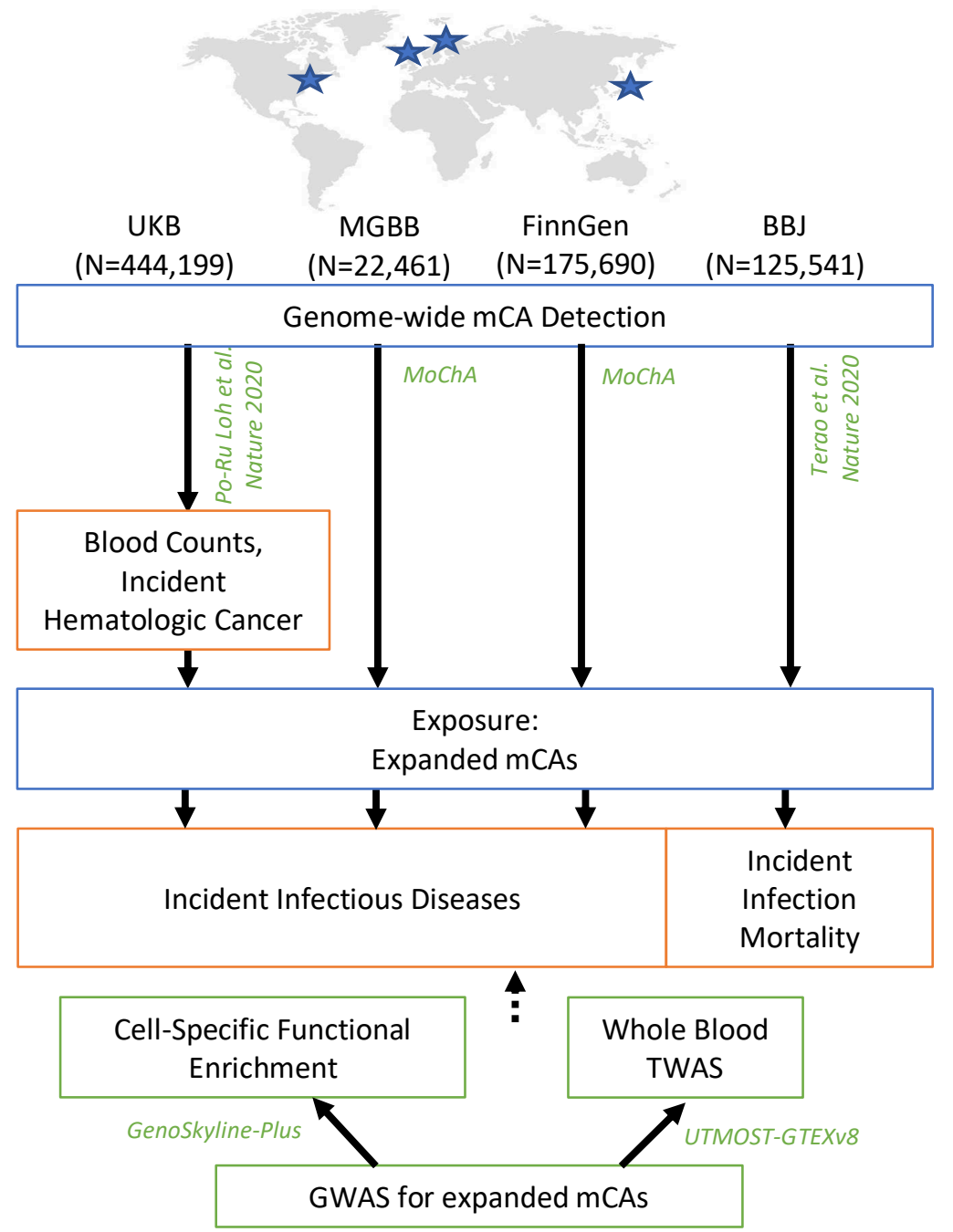

b.

All Expanded mCAs

(Cell fraction $>10 \%)$

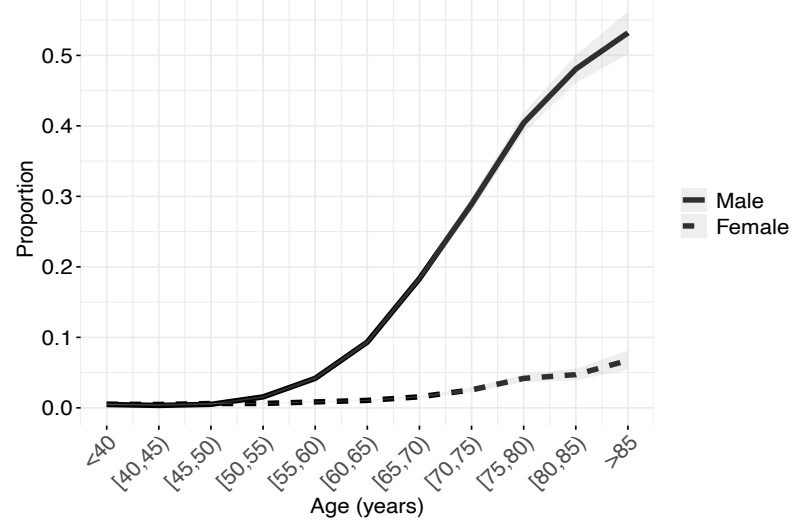

c.

Expanded Autosomal mCAs

(Cell fraction $>10 \%)$

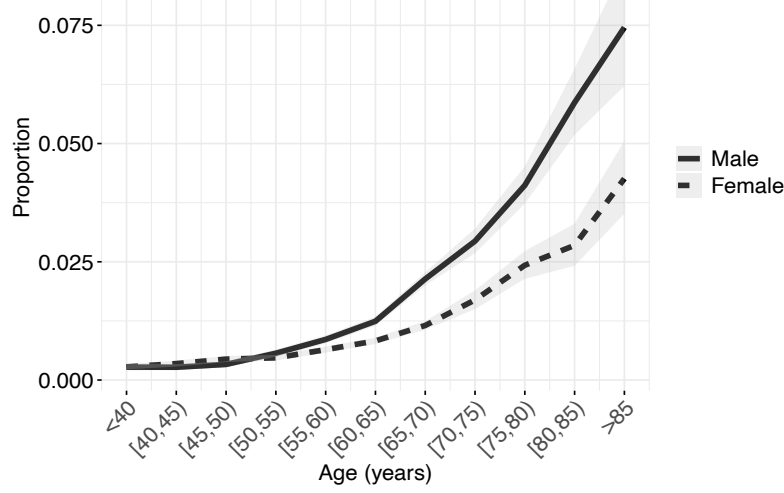

c.

All mCAs
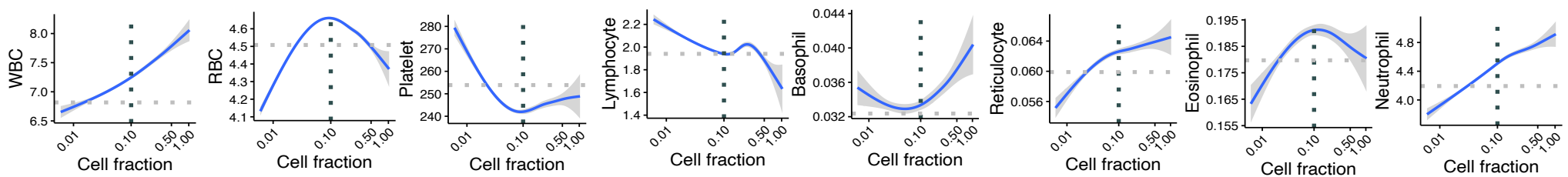

Autosomal mCAs
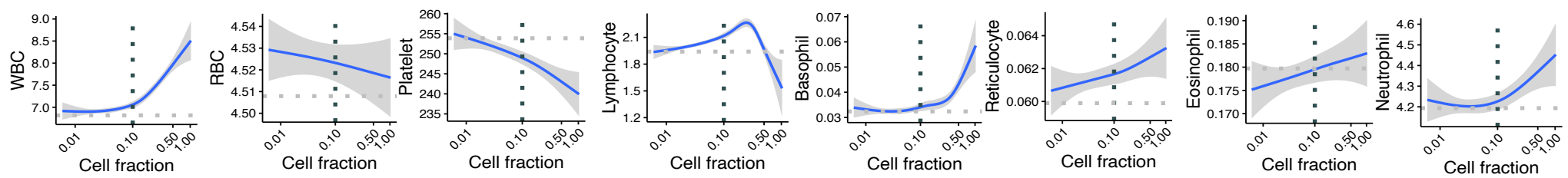

1 
2 Figure 1: Study schematic. a. Genome-wide mCAs were detected across the $\mathrm{UKB}^{4}$, MGBB (via 3 the MoChA pipeline), FinnGen (via the MoChA pipeline), and $\mathrm{BBJ}^{3}$. Association of expanded 4 mCAs (cell fraction $>10 \%$ ) with incident infectious diseases in UKB, MGBB, and FinnGen and 5 with incident infectious disease mortality in BBJ was performed. A GWAS for expanded mCAs 6 was then performed in the UKB to discover causal factors for expanded mCAs. Using the 7 GWAS results, cell-specific functional enrichment analyses were performed using GenoSkyline8 Plus, which combines epigenetic and transcriptomic annotations with GWAS summary statistics 9 to estimate the relative contribution of cell-specific functional markers to the GWAS results. 10 Additionally, to prioritize putative causal genes and pathways promoting the development of 11 expanded mCAs, whole blood TWAS was performed using UTMOST via GTEx v8. Association 12 of $\mathbf{b}$. all expanded mCAs with cell fraction $>10 \%$, and $\mathbf{c}$. all expanded autosomal mCAs, with 13 age using 5-year age bins stratified by sex among individuals in the UKB, MGBB, FinnGen, and 14 BBJ combined. Plots by cohort and across other mCA groupings are available in

15 Supplementary Note 7, 8. d. Associations of mCA cell fraction with blood counts (in units of $1610^{\wedge} 9$ cells/L) in the UKB among individuals without prevalent hematologic cancer at time of 17 blood draw for genotyping and cell count measurement. The dotted horizontal lines reflect the 18 mean blood count for individuals without an mCA. The dotted vertical lines at cell fraction of

190.10 represents the cutoff for the expanded mCA definition. Individuals with known hematologic 20 cancer at time of or prior to blood draw for genotyping were excluded.

$21 \quad$ BBJ = BioBank Japan, GTEx v8 = Genotype-Tissue Expression project version 8,

22 GWAS=genome-wide association study, $\mathrm{MGBB}=$ Mass General Brigham Biobank, $\mathrm{mCA}=$ 23 mosaic chromosomal alterations, MoChA = Mosaic Chromosomal Alterations software

24 (https://github.com/freeseek/mocha), TWAS = transcriptome-wide association study, UKB = UK 25 Biobank, UTMOST $=$ Unified Test for MOlecular SignaTures. 
a.

3 c.
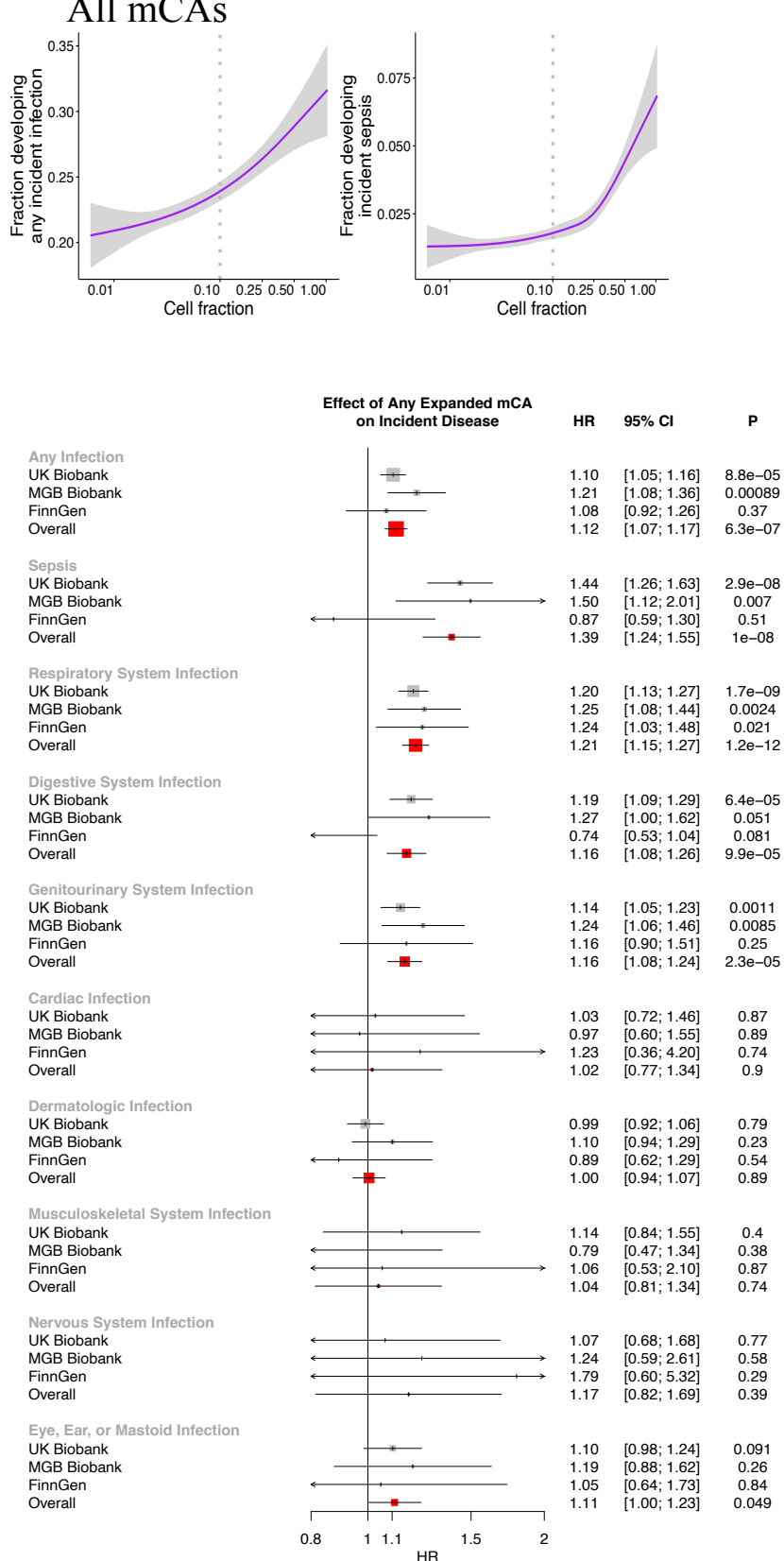

b. Autosomal mCAs
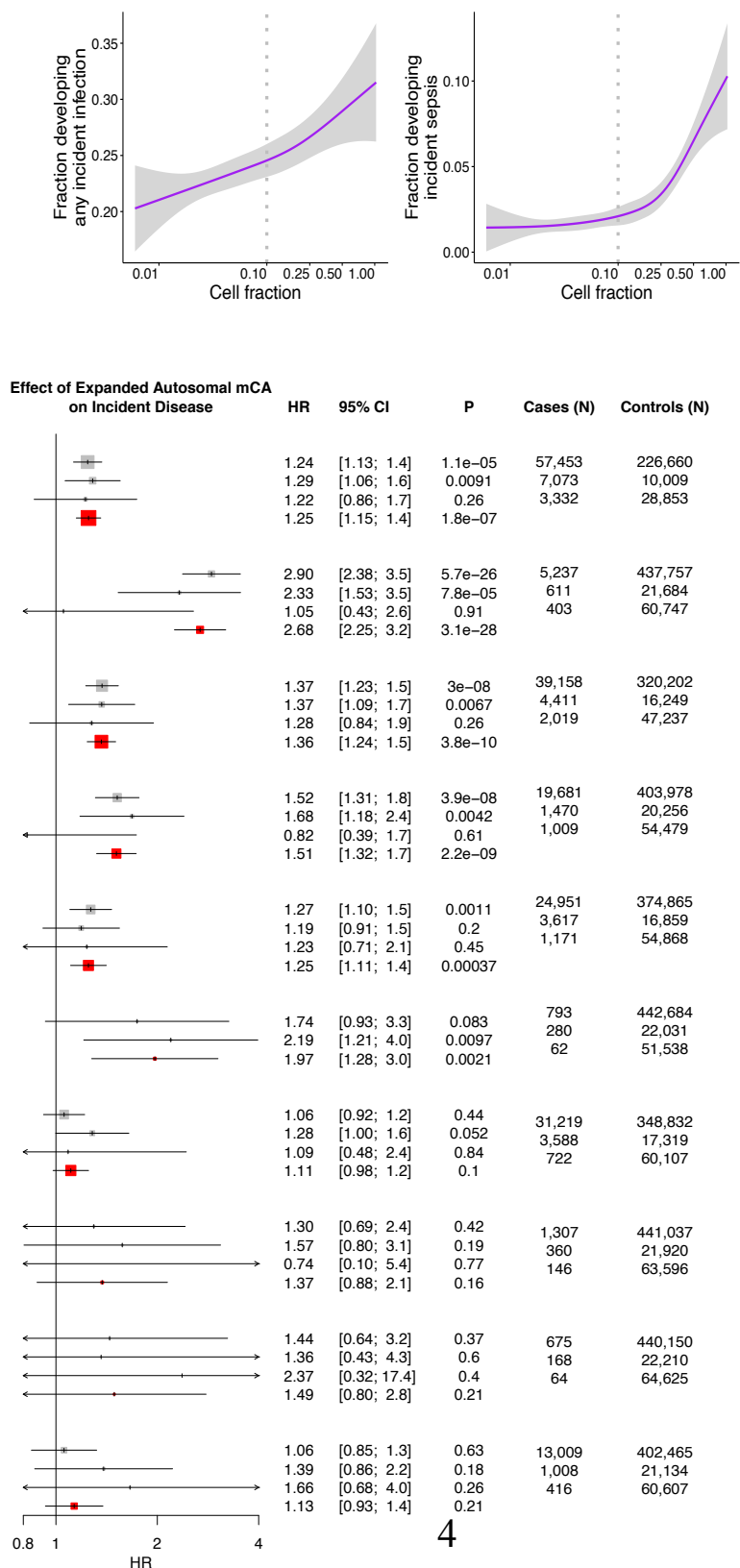

Figure 2: Associations of expanded mCAs with incident infections. Visualizing the dependence with cell fraction among a. all mCAs, and b. autosomal mCAs, of any incident infection and incident sepsis in the UKB among individuals without prevalent hematologic cancer at time of blood draw for genotyping across. The dotted vertical lines at cell fraction of 0.10 represents the cutoff for the expanded mCA definition. c. Association of all expanded mCAs, and separately, expanded autosomal mCAs with incident infections across individuals in the UKB, MGBB, and FinnGen. Analyses are adjusted for age, age ${ }^{2}$, sex, smoking status, and principal components 1-

1310 of ancestry. Individuals with prevalent hematologic cancer were excluded from analysis.

14 Association analyses for other groupings of mCAs (including across all mCAs regardless of cell 15 fraction, as well as chrX and chrY mCAs are provided in Supplementary Notes 10, 12). BBJ = 16 BioBank Japan, MGBB = Mass General Brigham Biobank, mCA = mosaic chromosomal 17 alterations, $\mathrm{UKB}=\mathrm{UK}$ Biobank. 
a.

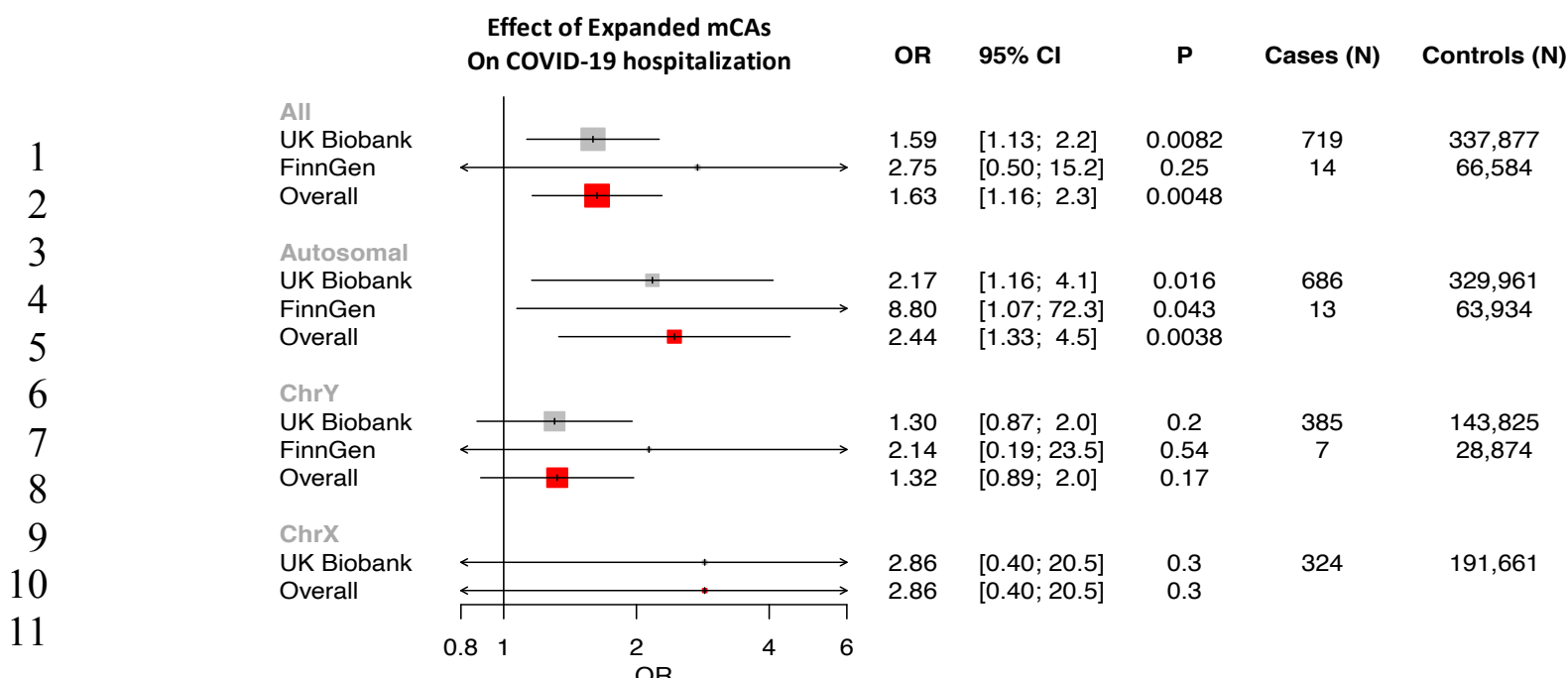

b.
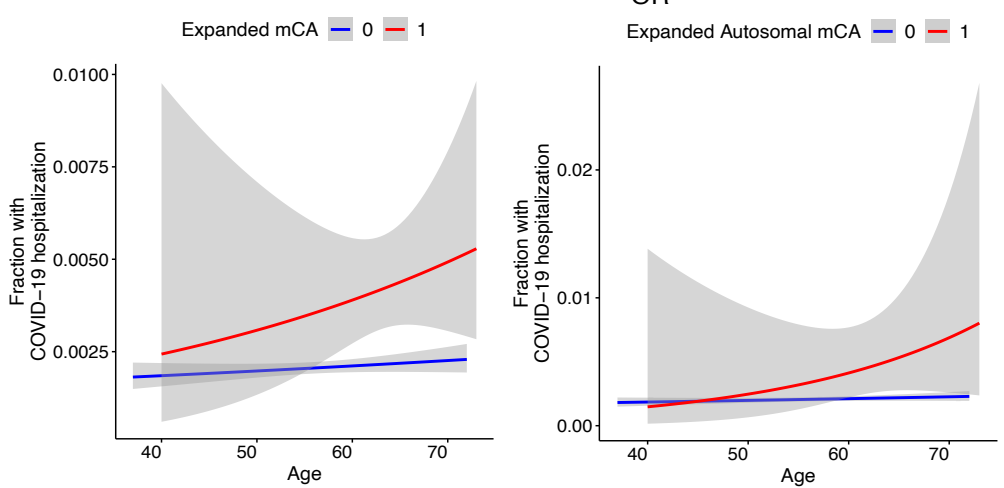

d.

C.

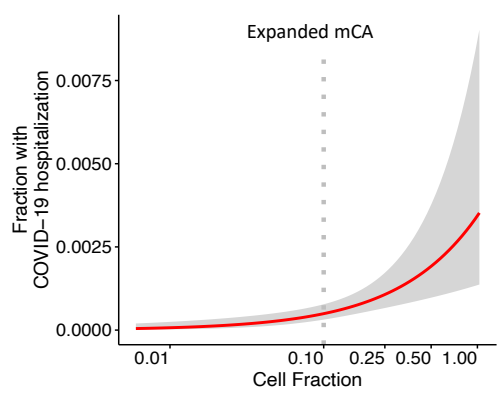

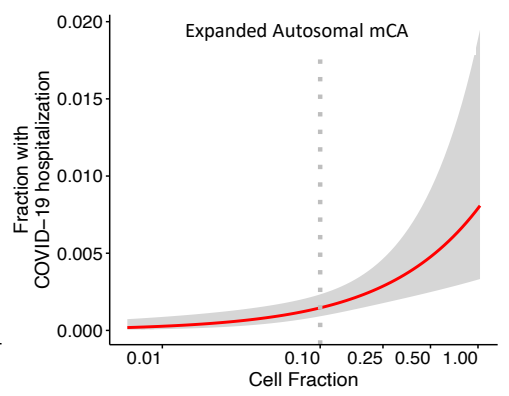

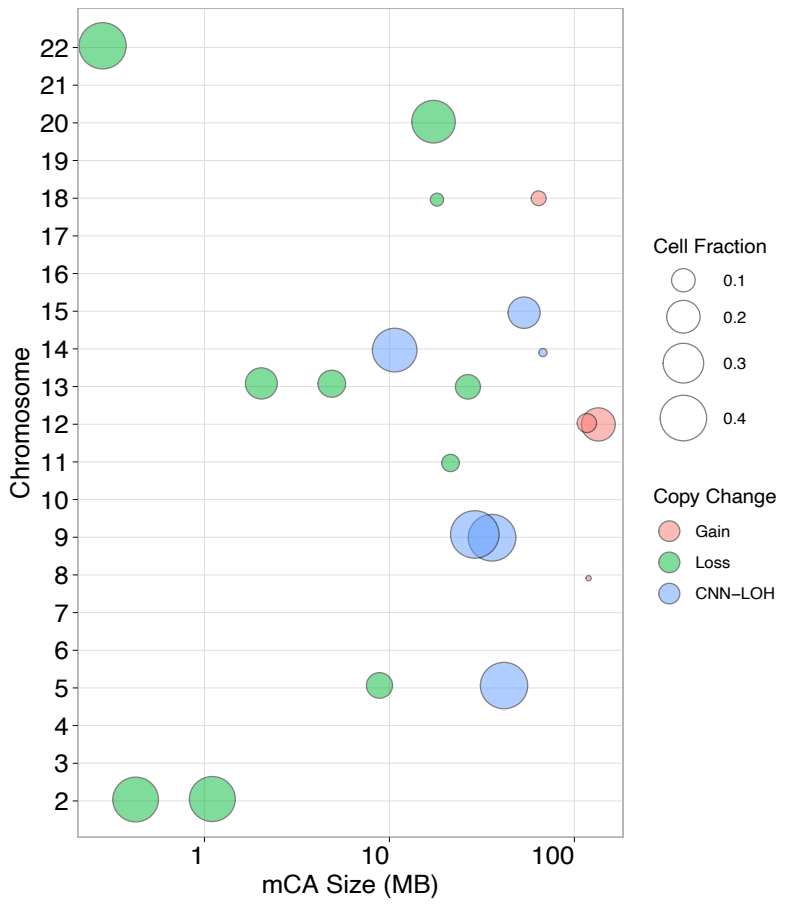

Figure 3: Association of expanded mCAs with COVID-19 Hospitalization. a. Associaiton of

14 expanded mCAs with COVID-19 Hospitalization across the UKB and FinnGen. Individuals with 15 known hematologic cancer at time of or prior to blood draw for genotyping were excluded.

16 Analyses are adjusted for age, age ${ }^{2}$, sex, ever smoking status, and principal components of

17 ancestry. b. Fraction of COVID-19 hospitalizations plotted by age, stratified by Expanded mCA

18 (left) and expanded autosomal mCA (right) c. Fraction of COVID-19 hospitalizations plotted by

19 cell fraction among expanded mCAs (left) and expanded autosomal mCAs (right).

20 d.Visualization of the diverse range of expanded autosomal mCAs detected across the genome

21 among individuals hospitalized with COVID-19 in the UK Biobank. Each point represents one

22 mCA carried by a case, with the $\mathrm{x}$-axis as the chromosome, $\mathrm{y}$-axis as the mCA size in mega-

23 bases of DNA (MB). Additional sensitivity analyses in the UKB are provided in Extended Data

24 Figure 8. $\mathrm{MGBB}=$ Mass General Brigham Biobank, UKB = UK Biobank, $\mathrm{MB}=$ megabase,

$25 \mathrm{CNN}-\mathrm{LOH}=$ copy number neutral loss of heterozygosity 

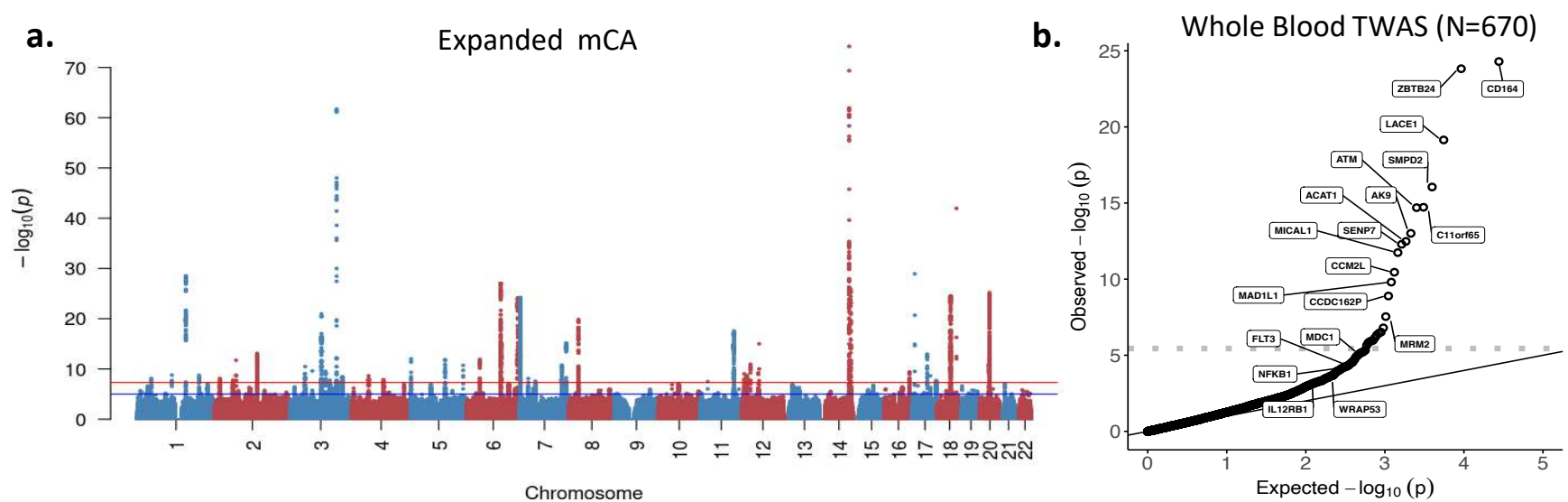

c.

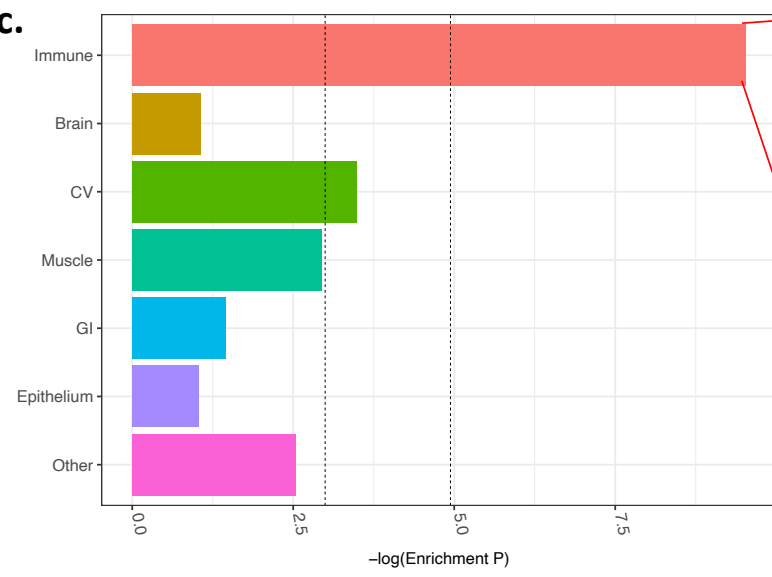

d.

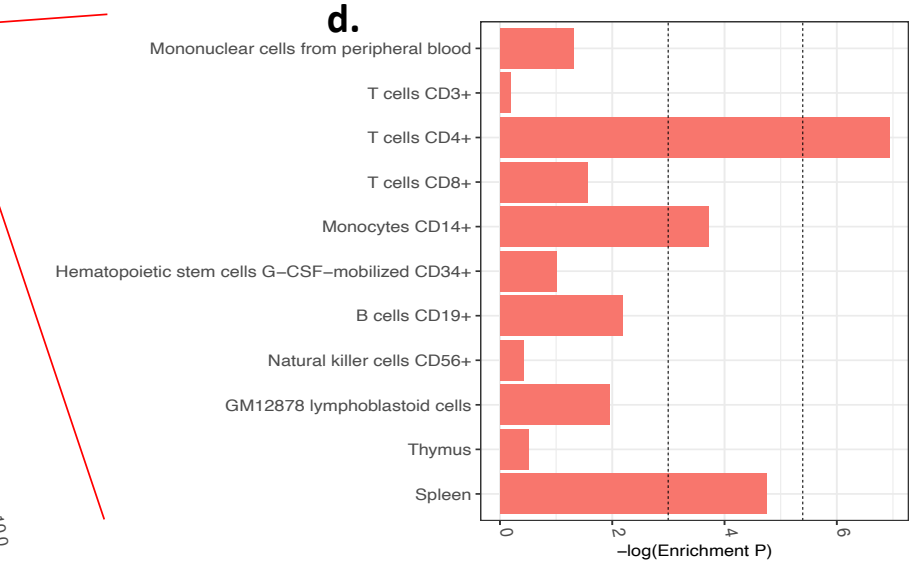

Figure 4: Inherited risk factors for expanded mCAs: GWAS, TWAS, and Cell Type Enrichment. a. GWAS for expanded mCA identified 63 independent loci. b. Quantile-quantile plot of the whole blood TWAS of the expanded mCA GWAS using 670 samples from GTExv8 shows enrichment across 62 genes. The horizontal dotted line reflects the Bonferroni-adjusted p-value for significance. Genes with TWAS $\mathrm{P}<5 \times 10^{-8}$ or those important in the pathway-enrichment analyses from Extended Data Figure 9 are labeled. c. cell-type enrichment results from the Expanded mCA GWAS across immune, brain, cardiovascular (CV), muscle, gastrointestinal (GI), epithelium, and other tissues as annotated using GenoSkyline-Plus annotations. D. Zooming in to show the stratified enrichment by specific categories of immune cells and tissues. Across panels C. and D., the vertical dotted lines indicate (1) $\mathrm{P}=0.05$ for suggestive enrichment, and (2) the Bonferroni-adjusted P-value for significant enrichment. GWAS = genome wide association study, TWAS = transcriptome-wide association study, $\mathrm{CV}=$ cardiovascular, $\mathrm{GI}=$ Gastrointestinal 
a) $\underline{M G B B}$

- Loss - $\mathrm{CN}-\mathrm{LOH}$ - Gain - Undetermined

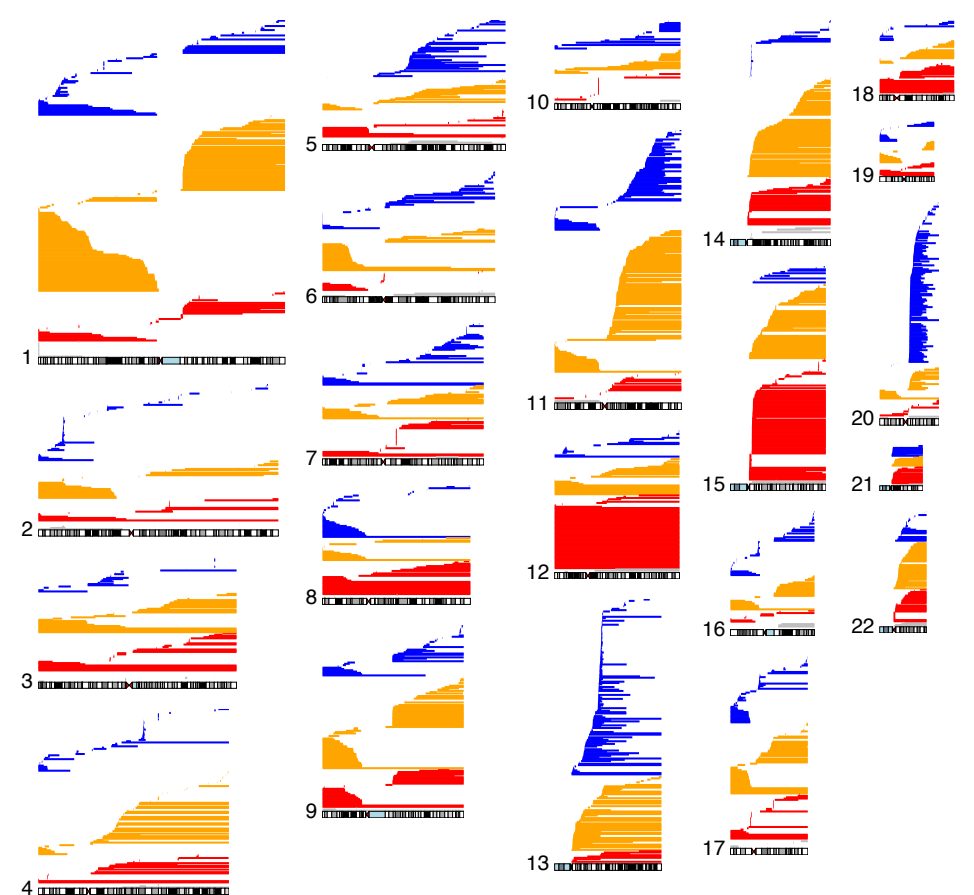

b) FinnGen

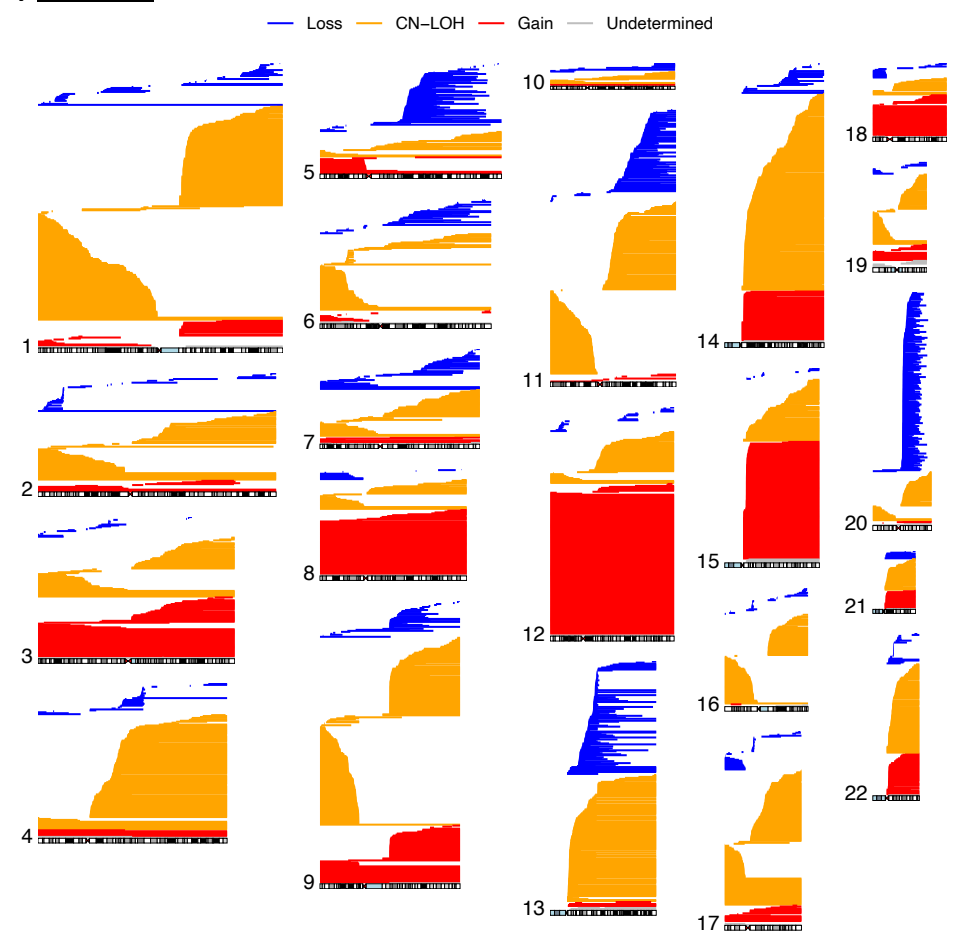

2 Extended Data Figure 1: mCA calls by chromosome in the MGBB and FinnGen, CN-LOH = 3 copy neutral loss of heterozygosity 4 


\begin{tabular}{|c|c|c|c|c|c|c|c|}
\hline & & $\begin{array}{l}\text { Effect of mCAs } \\
\text { plood counts (SD) }\end{array}$ & Beta (SD) & $95 \% \mathrm{Cl}$ & $\mathbf{P}$ & Participants (N) & Participants with $\mathrm{mCA}(\mathrm{N})$ \\
\hline \multicolumn{8}{|l|}{ All } \\
\hline White blood cell leukocyte count & & + & 0.32 & {$[0.31 ; 0.34]$} & $2.6 \mathrm{e}-227$ & 430,370 & 11,915 \\
\hline Neutrophill count & & + & 0.26 & {$[0.24 ; 0.28]$} & $3.9 e-151$ & 429,489 & 11,953 \\
\hline Monocyte count & & + & 0.25 & {$[0.23 ; 0.27]$} & $8 e-145$ & 427,354 & 11,899 \\
\hline Red blood cell erythrocyte count & $\leftarrow$ & & -0.19 & {$[-0.21 ;-0.18]$} & $2.5 e-113$ & 430,123 & 11,967 \\
\hline Lymphocyte count & & + & 0.13 & {$[0.11 ; 0.15]$} & $1.3 e-39$ & 429,366 & 11,751 \\
\hline Platelet count & & + & 0.11 & {$[0.10 ; 0.13]$} & $6.2 \mathrm{e}-31$ & 429,941 & 11,956 \\
\hline Basophill count & & + & 0.11 & {$[0.08 ; 0.13]$} & $3 e-24$ & 428,816 & 11,927 \\
\hline Eosinophill count & & + & 0.06 & {$[0.04 ; 0.08]$} & $1.6 \mathrm{e}-08$ & 406,400 & 11,737 \\
\hline Reticulocyte count & + & & -0.02 & {$[-0.04 ; 0.00]$} & 0.047 & 422,655 & 11,780 \\
\hline \multicolumn{8}{|l|}{ Autosomal } \\
\hline Lymphocyte count & & $\mp$ & 0.40 & {$[0.36 ; 0.44]$} & $4.2 \mathrm{e}-84$ & 429,366 & 2,657 \\
\hline White blood cell leukocyte count & & $\mp$ & 0.37 & {$[0.33 ; 0.41]$} & $5.5 e-75$ & 430,370 & 2,797 \\
\hline Basophill count & & $\mp$ & 0.14 & {$[0.09 ; 0.18]$} & $4.3 e-11$ & 428,816 & 2,850 \\
\hline Monocyte count & & $\mp$ & 0.12 & {$[0.08 ; 0.16]$} & $7.3 e-10$ & 427,354 & 2,821 \\
\hline Red blood cell erythrocyte count & $\mp$ & & -0.06 & {$[-0.10 ;-0.03]$} & 0.00012 & 430,123 & 2,858 \\
\hline Reticulocyte count & & $\mp$ & 0.07 & {$[0.03 ; 0.11]$} & 0.00032 & 422,655 & 2,834 \\
\hline Neutrophill count & & $\mp$ & 0.07 & {$[0.03 ; 0.11]$} & 0.00052 & 429,489 & 2,865 \\
\hline Platelet count & $\mp$ & & -0.05 & {$[-0.09 ;-0.02]$} & 0.0055 & 429,941 & 2,841 \\
\hline \multicolumn{8}{|l|}{ ChrY } \\
\hline White blood cell leukocyte count & & + & 0.29 & {$[0.27 ; 0.32]$} & $4.7 e-138$ & 198,676 & 8,875 \\
\hline Neutrophill count & & + & 0.28 & {$[0.26 ; 0.30]$} & $2.3 e-124$ & 198,290 & 8,848 \\
\hline Monocyte count & & + & 0.25 & {$[0.23 ; 0.28]$} & $3.2 \mathrm{e}-101$ & 197,945 & 8,839 \\
\hline Platelet count & & + & 0.22 & {$[0.19 ; 0.24]$} & $8.8 \mathrm{e}-74$ & 198,487 & 8,873 \\
\hline Red blood cell erythrocyte count & + & & -0.15 & {$[-0.18 ;-0.13]$} & $1.1 \mathrm{e}-39$ & 198,545 & 8,865 \\
\hline Lymphocyte count & & + & 0.11 & {$[0.09 ; 0.13]$} & $4.3 e-21$ & 198,198 & 8,844 \\
\hline Basophill count & & + & 0.08 & {$[0.06 ; 0.11]$} & $1.1 \mathrm{e}-11$ & 197,901 & 8,837 \\
\hline Eosinophill count & & $\mp$ & 0.05 & {$[0.03 ; 0.07]$} & $1.6 \mathrm{e}-05$ & 197,711 & 8,828 \\
\hline \multicolumn{8}{|l|}{ ChrX } \\
\hline White blood cell leukocyte count & & r & 0.24 & {$[0.12 ; 0.36]$} & $7.6 e-05$ & 231,694 & 386 \\
\hline Lymphocyte count & & $\longrightarrow$ & 0.18 & {$[0.07 ; 0.30]$} & 0.0024 & 231,168 & 381 \\
\hline Monocyte count & & $\longrightarrow$ & 0.18 & {$[0.06 ; 0.30]$} & 0.0036 & 229,409 & 383 \\
\hline Neutrophill count & & 1 & 0.18 & {$[0.06 ; 0.30]$} & 0.0036 & 231,199 & 385 \\
\hline \multirow{3}{*}{ Platelet count } & & $\ldots$ & 0.16 & {$[0.04 ; 0.28]$} & 0.0083 & 231,454 & 386 \\
\hline & 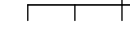 & \begin{tabular}{l|l|l|l|} 
& 1 &
\end{tabular} & & & & & \\
\hline & $-0.2-0.1 \quad 0$ & $\begin{array}{lll}0 & 0.1 & 0.25\end{array}$ & 0.5 & & & & \\
\hline
\end{tabular}

5 Extended Data Figure 2: Association of blood counts with expanded mCAs. Associations are 6 adjusted for age, age ${ }^{2}$, sex, smoking status, and principal components of ancestry. $\mathrm{mCA}=$ mosaic 7 chromosomal alterations. 
a.

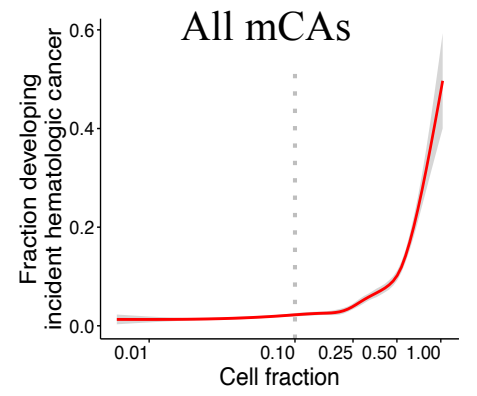

c.

Hematologic Cancer Hematologic Cance CLL Any Cancer Malignant Cance MPN

Lung Cancer Myeloid Leukemia Epithelial Cancer Colorectal Cancer Bkin Cancer Nervous System Cancer Bladder Cancer Carcinoma in situ Cancer ConnectiveTissue Cance

Autosomal Hematologic Cancer Lymphoid Leukemia MPL

Any Cancer

Any Cancer Myeloid Leukemia Skin Cancer Lung Cancer Benign Cancer Colorectal Cancer Carcinoma in situ Cancer Cancer Bpithelial Cancer Nervous System Cancer Bladder Cancer Prostate Cancer

ChrY Benign Cancer Prostate Cancer Malignant Cancer MLL

Epithelial Cancer Myeloid Leukemia MPN Carcinoma in situ Cancer Skin Cancer Colorectal Cancer Colorectal Cancer ConnectiveTissue Cance Bladder Cancer

Hematologic Cancer CLL Lymphoid Leukemia Malignant Cancer Epithelial Cancer Any Cancer Skin Cancer Breast Cancer Lung Cancer Carcinoma in situ Cancer

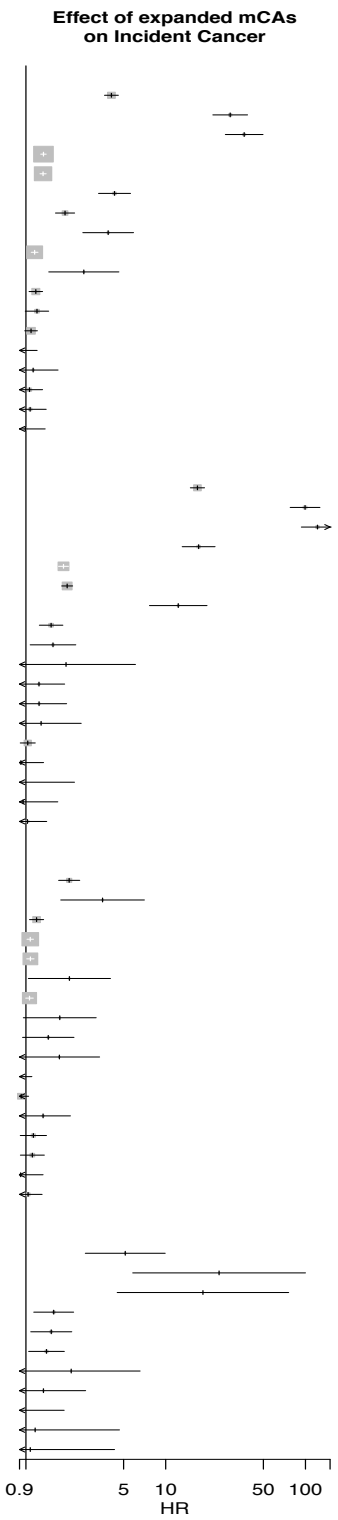
Benign Cancer Prostate Cancer

Lymphoid Leukemia

b.

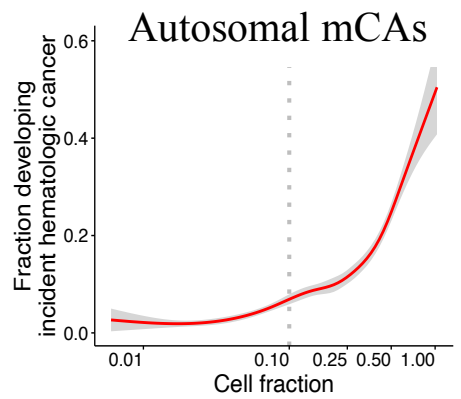

HR $95 \%$

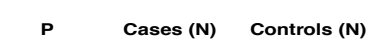

$4.09 \quad[3.65 ; 4.6]$

2.6e-132

$\begin{array}{ccc}36.44 & {[26.75 ; 49.6]} & 4.3 \mathrm{e}-115 \\ 1.34 & {[1.28 ; 1.4]} & 8.8 \mathrm{e}-38\end{array}$

$\begin{array}{ll}1.28 ; 1.4] & 8.8 \mathrm{e}-38 \\ 1.26 ; 1.4] & 1.1 \mathrm{e}-28\end{array}$

3.31; 5 .

$\begin{array}{ll}1.31 ; 5.6] & 9.6 \mathrm{e}-28 \\ 1.63 ; 2.2] & 6.1 \mathrm{e}-16\end{array}$

$2.56 ; 5.9$

$1.8 \mathrm{e}-10$

$1.46 ; 4.6]$

(1.05; 1.4$]$

$\left[\begin{array}{ll}0.99 ; & 1.4 \\ 0.98 ; & 1.2\end{array}\right]$

$[0.60 ; 1.2]$

$[0.75 ; 1.7]$

$[0.86 ; 1.3]$

$[0.82 ; 1.4]$

0.062

0.11
0.36
0.57

0.57
0.59
0.61

732
$.59-1,573$

$\begin{array}{ll}0.61 & 2,499 \\ 0.96 & 959\end{array}$

$3,744 \quad 440,455$

$\begin{array}{ll}342 & 443,794 \\ 543,857\end{array}$

$\begin{array}{ll}38,346 & 387,421 \\ 605,853\end{array}$

$\begin{array}{ll}650 & 443,366 \\ 2,619 & 441,145\end{array}$

$\begin{array}{ll}2,619 & 441,145 \\ 255 & 443,944\end{array}$

$36,419 \quad 407,780$

$280 \quad 443,919$

$4,463 \quad 435,576$
5,378

$\begin{array}{ll}9,396 & 422,188 \\ 5,378 & 428,551\end{array}$

428,551

441,315

441,700
443,240

$\begin{array}{lllll}16.87 & {[15.03 ; 18.9]} & 0 & 3,744 & 440,455\end{array}$

$\begin{array}{ll}99.35 & {[77.86 ; 126.8]} \\ 121.92 & {[93.56 ; 158.9]}\end{array}$

$\begin{array}{ll}17.21 & {[93.56 ; 158.9} \\ 1.86 & {[1.14 ; 22.5]}\end{array}$

$\begin{array}{ccc}3.3 e-95 & 650 & 443,366 \\ 3.3 e-51 & 56,778 & 387,421 \\ 7.6 e-51 & 38,346 & 405,853\end{array}$

$2.3 e-25 \quad 255 \quad 405,853$

$\begin{array}{lll}2.3 e-05 & 9,396 & 443,944 \\ 0.02 & 2,619 & 42,188\end{array}$

$\begin{array}{lll}0.02 & 2,619 & 441,145 \\ 0.26 & 280 & 443,919\end{array}$

$\begin{array}{lll}0.32 & 280 & 443,919 \\ 0.32 & 2,463 & 439,230\end{array}$

$\begin{array}{lll}0.35 & 2,499 & 441,700\end{array}$

$\begin{array}{lcc}0.46 & 959 & 443,240 \\ 0.63 & 36,419 & 407,780\end{array}$

$\begin{array}{lll}0.66 & 5,378 & 428,551\end{array}$

$\begin{array}{lcl}0.71 & 732 & 443,467 \\ 0.87 & 1,573 & 441,315\end{array}$

$[0.63 ; 1.3]$

$[0.31 ; 2.2]$

$[0.75 ; 1.4]$

435,576

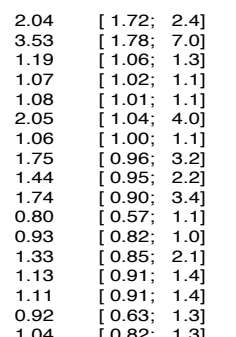

$\begin{array}{ccc}5.7 \mathrm{e}-16 & 1,402 & 202,938 \\ 0.00032 & 104 & 204,475 \\ 0.0027 & 4,784 & 195,961 \\ 0.0072 & 28,582 & 175,997 \\ 0.014 & 19,983 & 184,596 \\ 0.038 & 219 & 204,360 \\ 0.062 & 18,308 & 186,271 \\ 0.068 & 149 & 204,430 \\ 0.088 & 357 & 204,095 \\ 0.1 & 250 & 204,329 \\ 0.17 & 883 & 203,696 \\ 0.21 & 5,073 & 193,754 \\ 0.22 & 347 & 204,232 \\ 0.26 & 1,362 & 201,801 \\ 0.29 & 2,113 & 202,466 \\ 0.64 & 590 & 203,989 \\ 0.76 & 1,177 & 202,432\end{array}$

$\begin{array}{cc}5.14 & {[2.66 ; 9.9]} \\ 24.10 & {[5.82 ; 99.9]} \\ 18.47 & {[4.50 ; 75.7]} \\ 1.58 & {[1.14 ; 2.2]} \\ 1.52 & {[1.08 ; 2.1]} \\ 1.40 & {[1.05 ; 1.9]} \\ 2.11 & {[0.68 ; 6.5]} \\ 1.33 & {[0.67 ; 2.7]} \\ 0.78 & {[0.32 ; 1.9]} \\ 1.16 & {[0.29 ; 4.7]} \\ 1.07 & {[0.27 ; 4.3]}\end{array}$

$\begin{array}{ccc}1 \mathrm{e}-06 & 1,631 & 237,989 \\ 1.1 \mathrm{e}-05 & 123 & 239,497 \\ 5.1 \mathrm{e}-05 & 155 & 239,465 \\ 0.0061 & 18,363 & 221,257 \\ 0.015 & 18,111 & 221,509 \\ 0.023 & 28,196 & 211,424 \\ 0.2 & 1,101 & 237,429 \\ 0.42 & 4,323 & 228,434 \\ 0.58 & 5,328 & 224,083 \\ 0.83 & 1,217 & 238,207 \\ 0.92 & 1,616 & 238,004\end{array}$

Extended Data Figure 3: Association of mCAs with incident cancer in the UK Biobank.

Association of a) all mCA and b) autosomal mCA cell fraction with incident hematologic cancer. The dotted vertical line at cell fraction of 0.1 shows the cutoff point for expanded mCAs (defined as mCAs with cell fraction $>10 \%$ ). c) Association of expanded mCA categories ( with cell fraction $>10 \%$ ) with incident cancer in the UK Biobank. Analyses are adjusted for age, age ${ }^{2}$, sex, smoking status, and principal components of ancestry. Individuals with a history of hematologic

44

45 cancer at enrollment were removed from analysis. $\mathrm{CLL}=$ chronic lymphocytic leukemia, MPN = 46 
a.

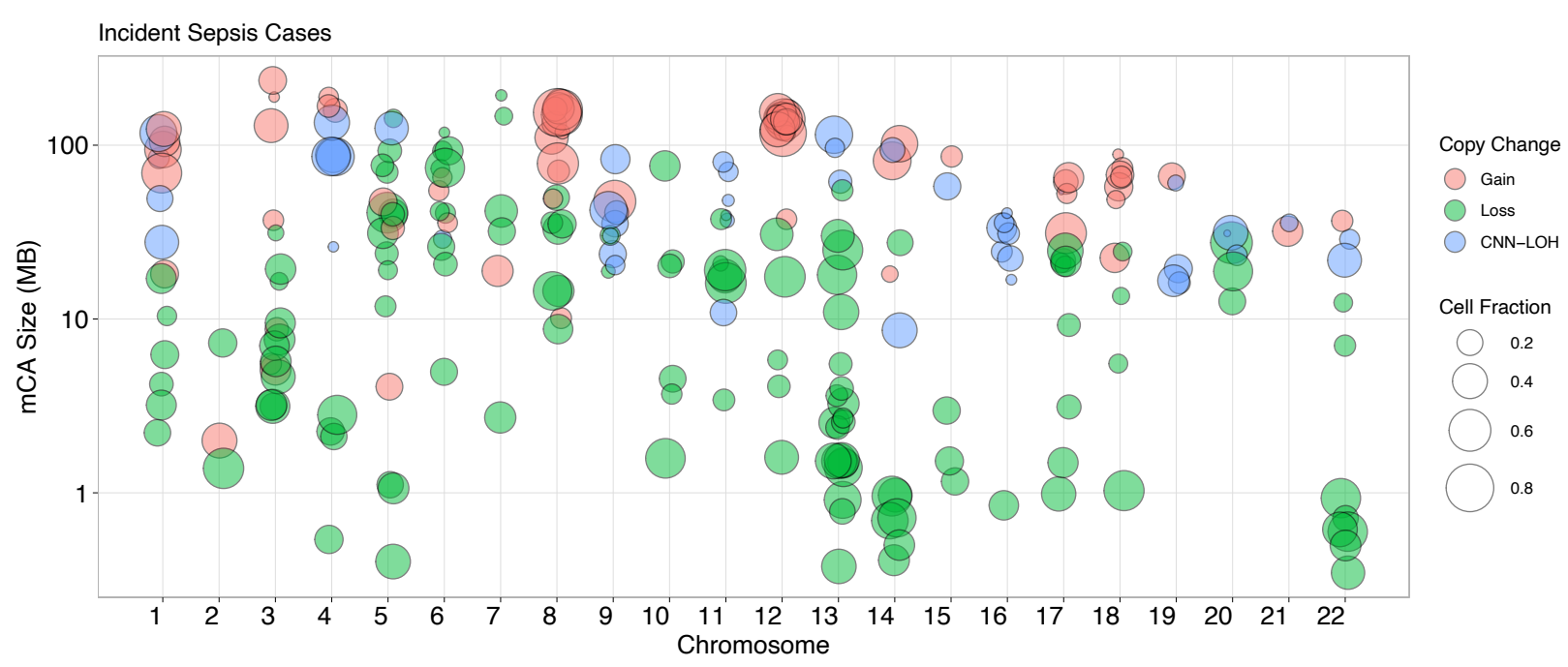

b.

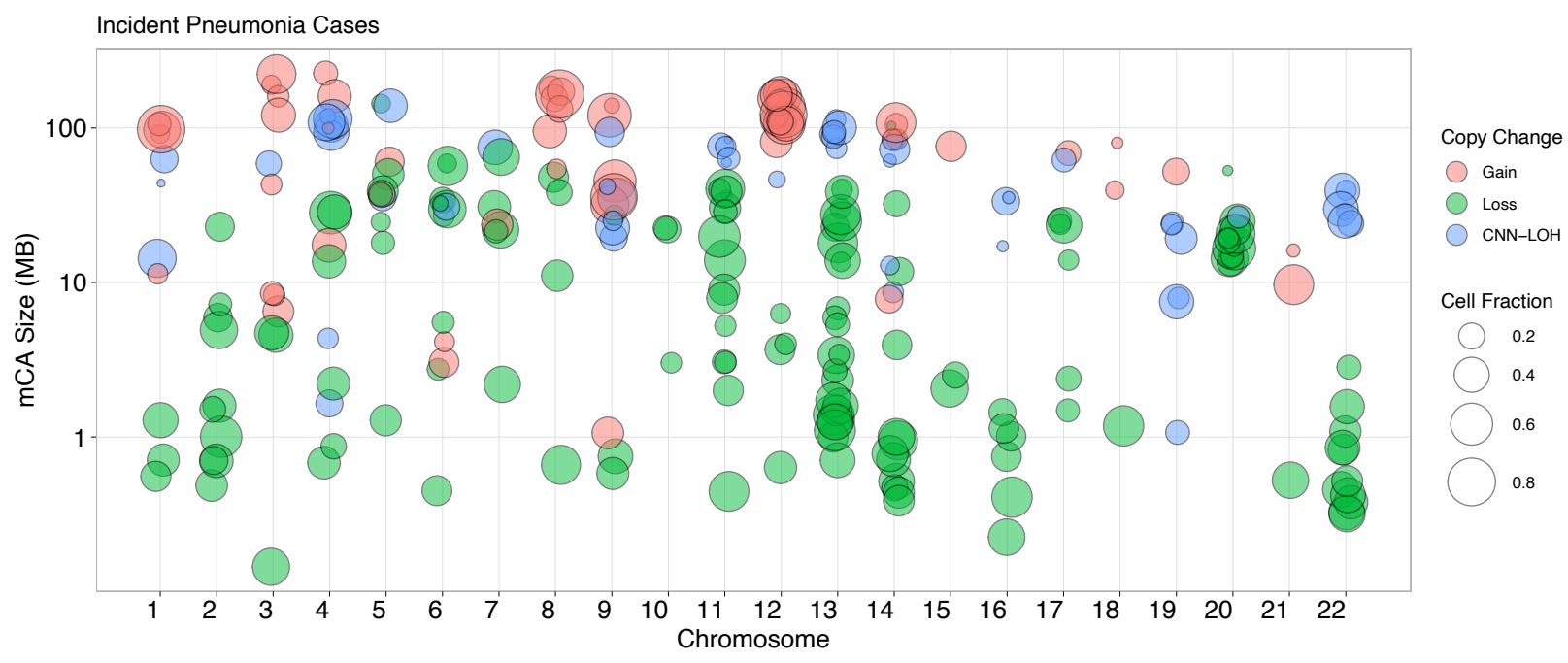

1

Extended Data Figure 4: Visualization of the diverse range of expanded autosomal mCAs detected across the genome among individuals with $\mathrm{a}$. incident sepsis and $\mathrm{b}$. incident pneumonia in the UKB. Each point represents one mCA carried by a case, with the $\mathrm{x}$-axis as the chromosome, $y$-axis as the mCA size in mega-bases of DNA (MB), color as the copy change, and size of the point as the cell fraction of that mCA. CNN-LOH=copy number neutral loss of heterozygosity, $\mathrm{MB}=$ megabases of $\mathrm{DNA}, \mathrm{mCA}=$ mosaic chromosomal alterations 
a.

Effect of Autosomal mCAs
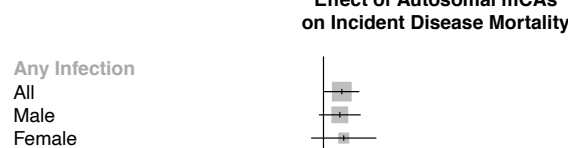

Nervous System Infection

All

Male

Respiratory System Infection

All

Male

Female

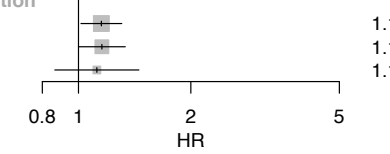

b.

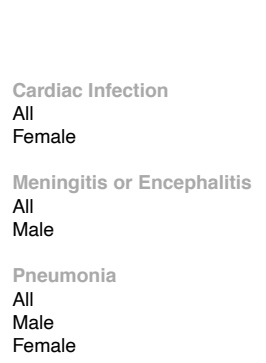

Effect of Autosomal mCAs on Incident Disease Mortality $\quad \mathrm{HR} \quad 95 \% \mathrm{Cl}$

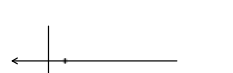

$1.11 \quad[0.56 ; 2.2]$

$\mathbf{P}$

Cases (N)

Controls (N)

Cases with mCA (N)

Controls with mCA (N)

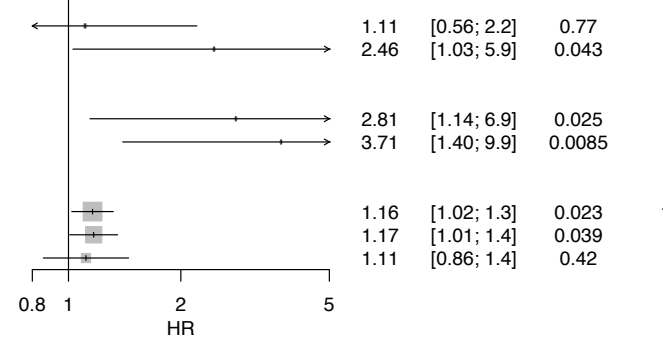

50
25

72,317

11

10,485
4,187

$21 \quad 72,317$

$\begin{array}{ll}21 & 72,317 \\ 17 & 39,978\end{array}$

$1,299 \quad 72,317$

1,299
928
371

72,317
39,978
32,339

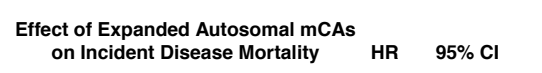

Sepsis
All
Male
Cases (N)

Controls (N)

Cases with mCA (N)

Controls with mCA (N)

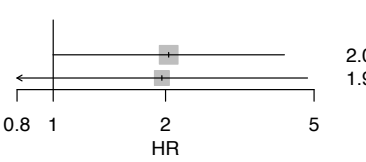

\begin{tabular}{llll} 
& {$[1.0 ; 4.2]$} & 0.05 & 27 \\
\hline $0.8 ; 4.8]$ & 0.14 & 16
\end{tabular}

72,317
39,978
753
471

Extended Data Figure 5: Suggestive associations $(\mathrm{P}<0.05)$ of mCAs with incident infectionrelated mortality in Biobank Japan. Associations of autosomal mCAs with a) organ-system level infections and b) specific infection categories. c) Association of expanded autosomal mCAs with Sepsis. Full results are in Supplementary Table 6. Associations are presented among individuals without any cancer history. $\mathrm{mCA}=$ mosaic chromosomal alterations. 


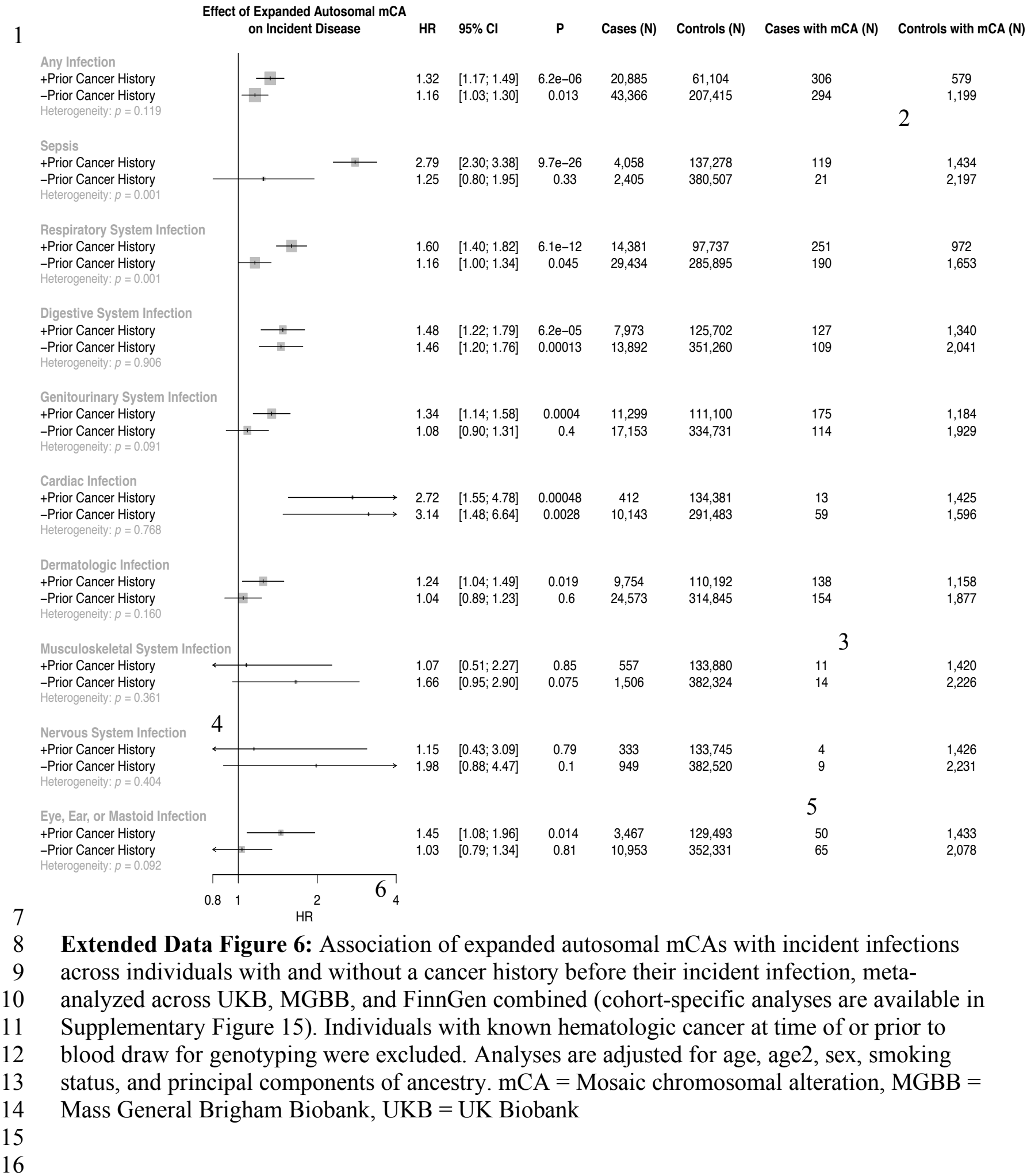


a.
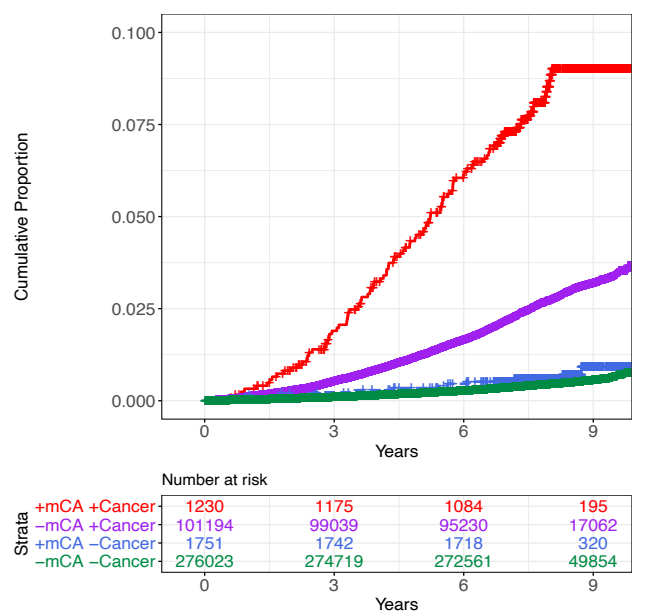

b.

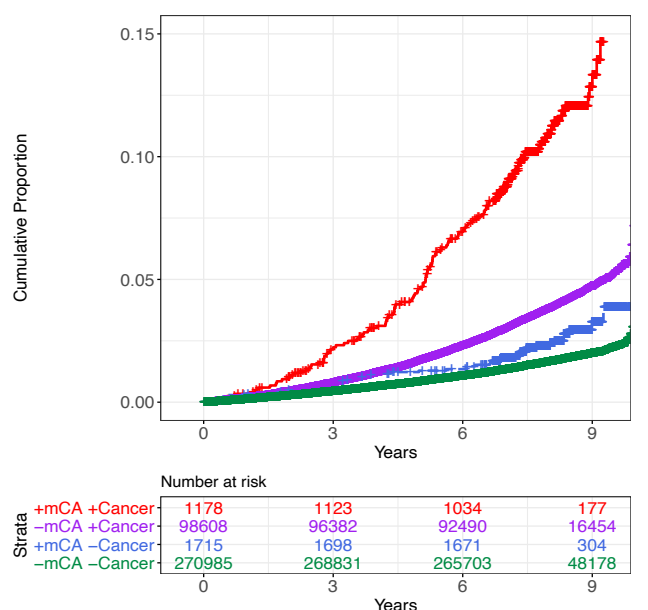

Years

c.

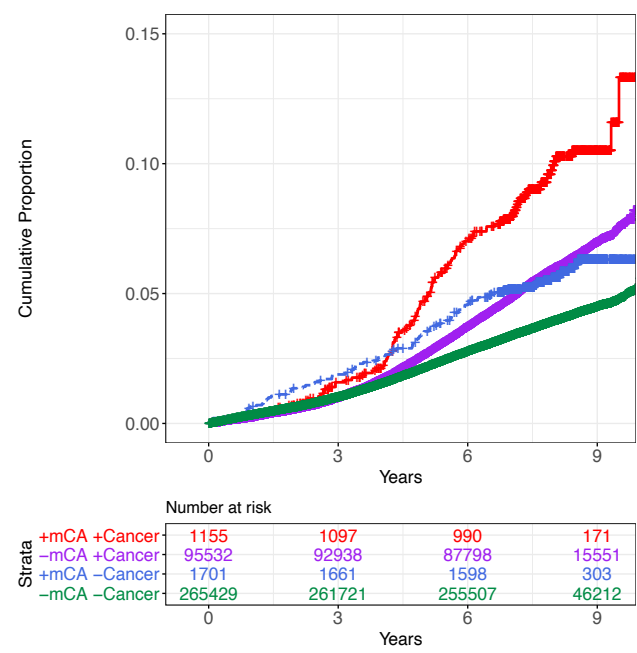

MGB Biobank
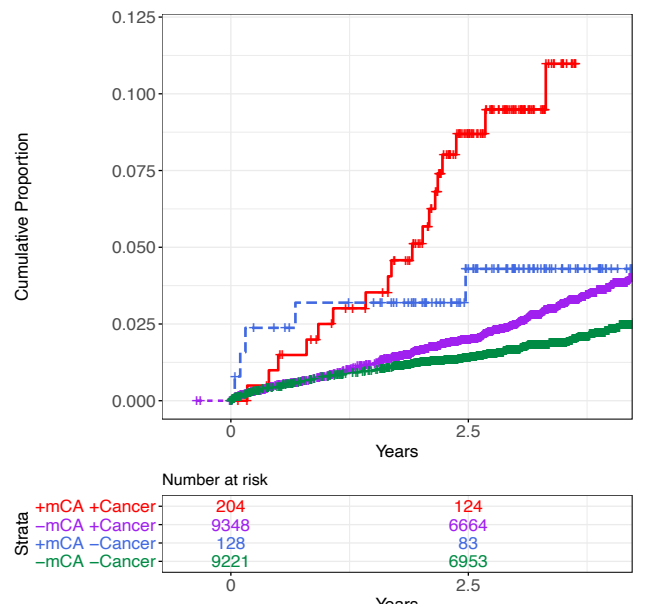

Years

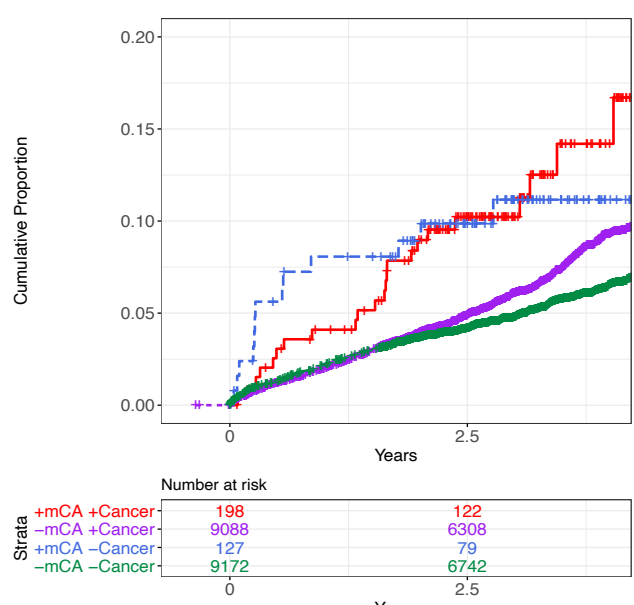

Years
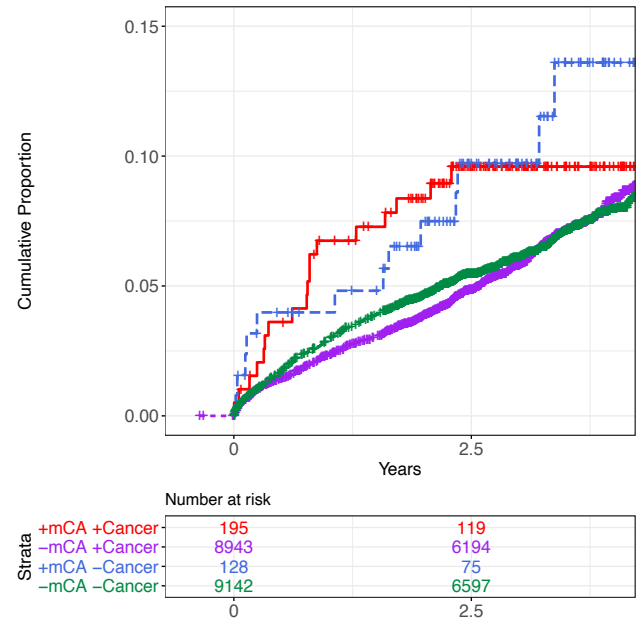

FinnGen
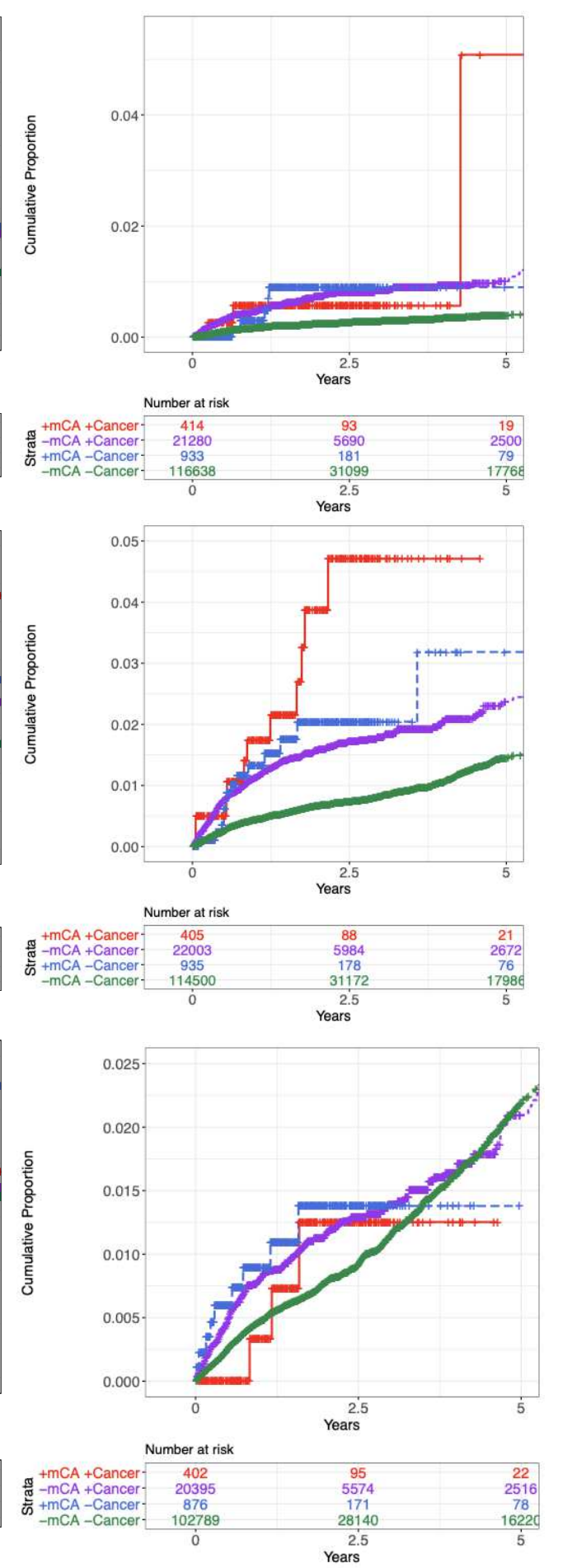

$1 \quad$ Extended Data Figure 7: Association of expanded autosomal mCAs with incident a. sepsis, $\mathbf{b}$.

2 pneumonia, and c. digestive system infection across carrier status for expanded autosomal mCAs

3 and any cancer diagnosis prior to the incident infection date. Individuals with known

4 hematologic cancer at time of or prior to blood draw for genotyping were excluded. $\mathrm{mCA}=$

5 mosaic chromosomal alterations. 
a.
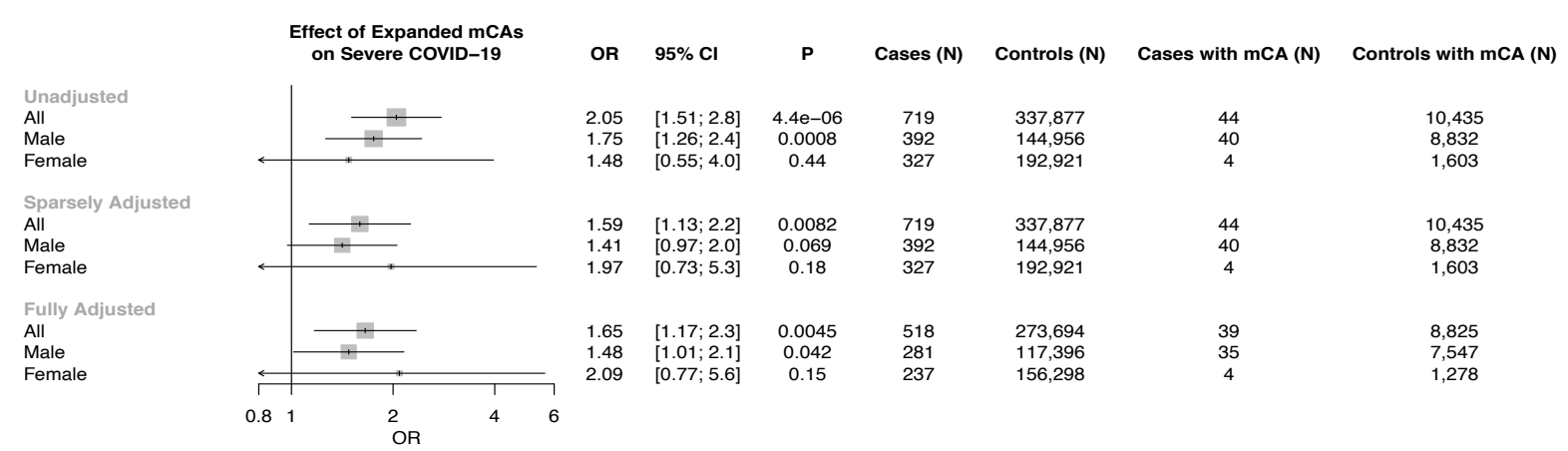

b.

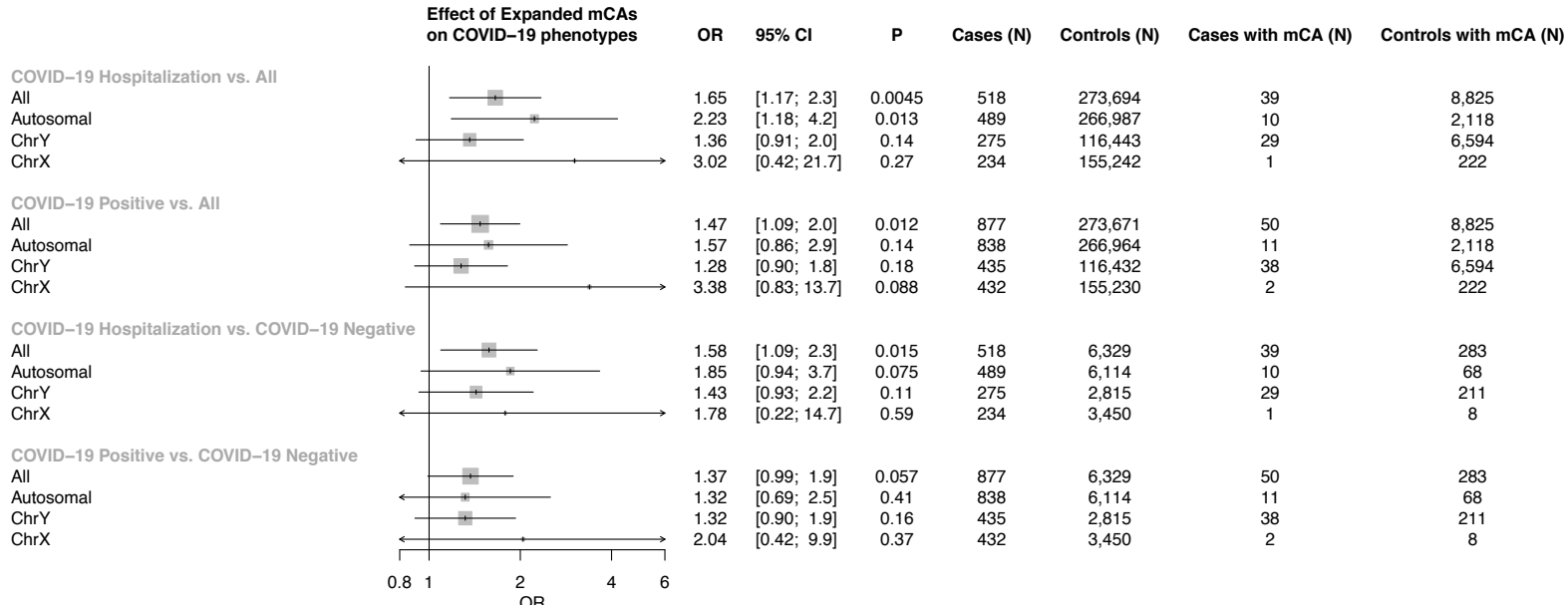

c.

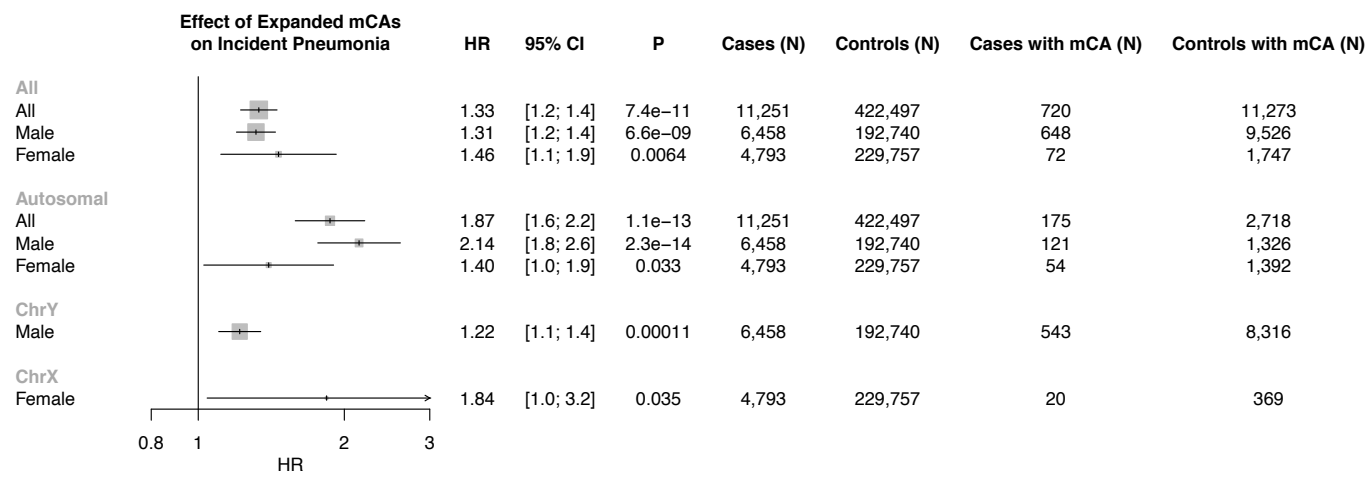

1 Extended Data Figure 8: Associations of expanded mCAs in the UK Biobank with COVID-19 and incident pneumonia. Associations of expanded mCAs with a. COVID-19 hospitalization across different adjustment models, and b. different COVID-19 phenotypes in a fully adjusted model. Adjustment models include 1) an unadjusted model, 2) a sparsely adjusted model which adjusts for age, age2, sex, smoking status, and principal components of ancestry, and 3) a fully adjusted model which additionally adjusts for Townsend deprivation index, BMI, and the following comorbidities: Asthma, COPD, CAD, T2D, any cancer, and HTN. mCA = mosaic 8 chromosomal alterations, $\mathrm{COPD}=$ chronic obstructive pulmonary disease, $\mathrm{CAD}=$ coronary 9 artery disease, T2D = type 2 diabetes mellitus. c. Association of expanded mCAs with incident 10 pneumonia stratified by sex, adjusted for age, age ${ }^{2}$, sex (in the All model only), smoking status, 11 and principal components of ancestry. $\mathrm{mCA}=$ mosaic chromosomal alterations 
a.

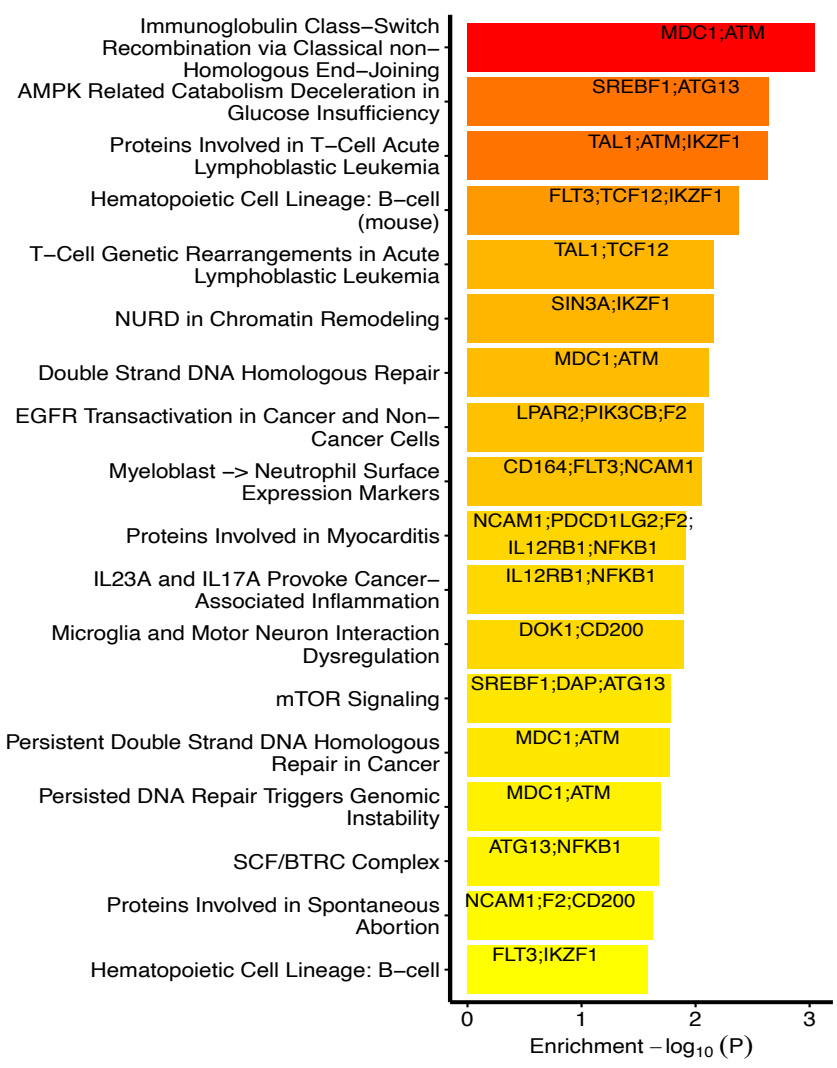
study b
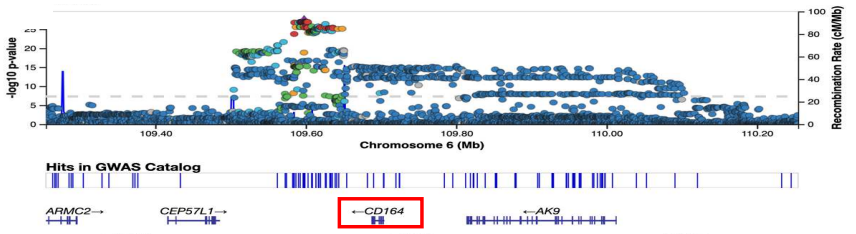

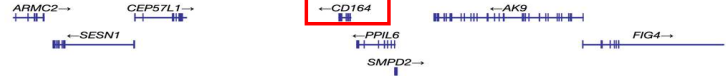

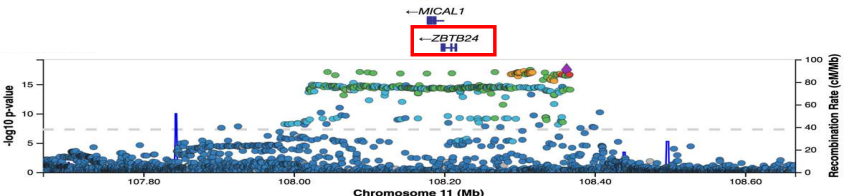

Hits in GWAS Catalog | || | ||||||||| | |||||| ||| | |
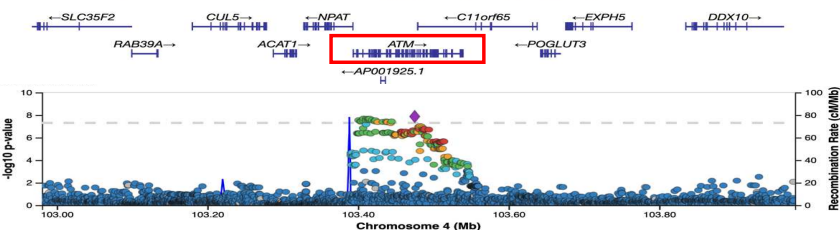

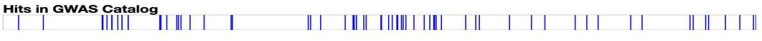
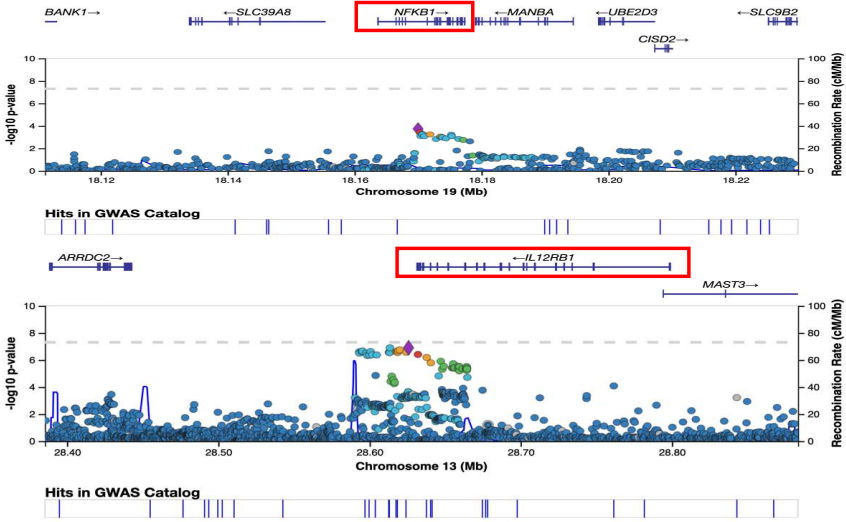

$\stackrel{P D X Y \rightarrow \sim U R A D}{\longrightarrow} \rightarrow$

CDX2
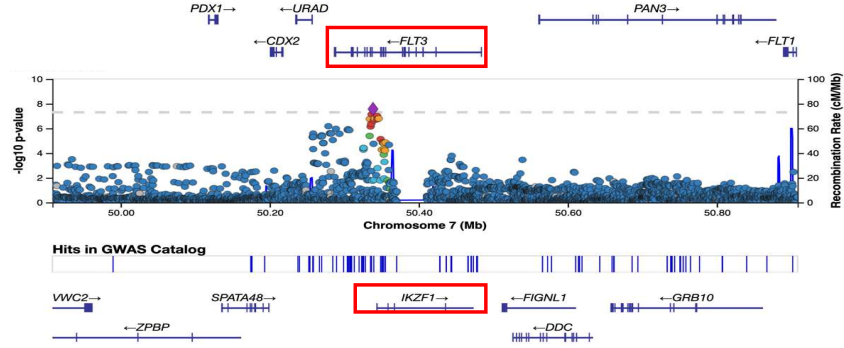

Extended Data Figure 9: Pathway enrichment of TWAS results using the Elsevier Pathways. a. Top results from pathway enrichment analysis of the TWAS results using the Elsevier Pathways. b. Highlighting the GWAS locus-zoom plots for some of the TWAS genes implicated in the top pathways from panel $b$. Red boxes highlight the gene(s) with strongest association in the TWAS analyses. GWAS = genome-wide association study, TWAS = transcriptome-wide association 


\begin{tabular}{|c|c|c|c|c|c|}
\hline & Adjustment & $H R$ & $P$ & $\begin{array}{l}\text { Lower } \\
95 \% C I\end{array}$ & $\begin{array}{c}\text { Upper } \\
95 \% C I\end{array}$ \\
\hline \multirow{6}{*}{ Sepsis } & Chemotherapy & 2.48 & $3.04 \mathrm{E}-17$ & 2.01 & 3.06 \\
\hline & Neutropenia & 1.65 & $3.98 \mathrm{E}-06$ & 1.33 & 2.04 \\
\hline & Aplastic anemia & 2.58 & $1.84 \mathrm{E}-18$ & 2.09 & 3.19 \\
\hline & Decreased white blood cell count & 1.65 & $3.98 \mathrm{E}-06$ & 1.33 & 2.04 \\
\hline & Bone marrow or stem cell transplant & 2.77 & $3.25 \mathrm{E}-21$ & 2.24 & 3.42 \\
\hline & Effects radiation NOS & 2.84 & $2.85 \mathrm{E}-22$ & 2.30 & 3.51 \\
\hline \multirow{6}{*}{ Pneumonia } & Chemotherapy & 2.11 & $1.47 \mathrm{E}-14$ & 1.74 & 2.55 \\
\hline & Neutropenia & 1.99 & $1.38 \mathrm{E}-12$ & 1.65 & 2.41 \\
\hline & Aplastic anemia & 2.16 & $2.17 \mathrm{E}-15$ & 1.79 & 2.62 \\
\hline & Decreased white blood cell count & 1.99 & $1.38 \mathrm{E}-12$ & 1.65 & 2.41 \\
\hline & Bone marrow or stem cell transplant & 2.20 & $5.04 \mathrm{E}-16$ & 1.82 & 2.66 \\
\hline & Effects radiation NOS & 2.27 & $2.59 \mathrm{E}-17$ & 1.88 & 2.75 \\
\hline
\end{tabular}

Extended Table 1: Sensitivity analysis of expanded autosomal mCA with incident sepsis and with pneumonia association in the UK Biobank among those with cancer prior to incident infection, separately adjusting for chemotherapy, neutropenia, aplastic anemia, decreased white blood cell count, bone marrow or stem cell transplant, and radiation effects prior to infection (as defined using the Vanderbilt ICD-10 and ICD-9 phecode groupings ${ }^{10}$ ). Other covariates in the model included age, age ${ }^{2}$, sex, smoking status, and PC1-10 of ancestry. The summary stats (HR,

10 P-value, 95\% CI) reflect those for the expanded autosomal mCA term in each model.

$11 \mathrm{CI}=$ confidence interval; $\mathrm{HR}=$ hazard ratio; $\mathrm{mCA}=$ mosaic chromosomal alteration; $\mathrm{N}=$ 12 number; NOS $=$ not otherwise specified

13 


\section{References}

1 Gardner, I. D. The effect of aging on susceptibility to infection. Rev Infect Dis 2, 801810, doi:10.1093/clinids/2.5.801 (1980).

2 Gavazzi, G. \& Krause, K. H. Ageing and infection. Lancet Infect Dis 2, 659-666, doi:10.1016/s1473-3099(02)00437-1 (2002).

3 Terao, C. et al. Chromosomal alterations among age-related haematopoietic clones in Japan. Nature, doi:10.1038/s41586-020-2426-2 (2020).

4 Loh, P. R., Genovese, G. \& McCarroll, S. A. Monogenic and polygenic inheritance become instruments for clonal selection. Nature, doi:10.1038/s41586-020-2430-6 (2020).

5 Loh, P. R. et al. Insights into clonal haematopoiesis from 8,342 mosaic chromosomal alterations. Nature 559, 350-355, doi:10.1038/s41586-018-0321-x (2018).

6 Lin, S. H. et al. Mosaic chromosome Y loss is associated with alterations in blood cell counts in UK Biobank men. Sci Rep 10, 3655, doi:10.1038/s41598-020-59963-8 (2020).

7 Forsberg, L. A. et al. Mosaic loss of chromosome $\mathrm{Y}$ in peripheral blood is associated with shorter survival and higher risk of cancer. Nat Genet 46, 624-628, doi:10.1038/ng.2966 (2014).

8 Jacobs, K. B. et al. Detectable clonal mosaicism and its relationship to aging and cancer. Nat Genet 44, 651-658, doi:10.1038/ng.2270 (2012).

9 Laurie, C. C. et al. Detectable clonal mosaicism from birth to old age and its relationship to cancer. Nat Genet 44, 642-650, doi:10.1038/ng.2271 (2012).

10 Loftfield, E. et al. Predictors of mosaic chromosome Y loss and associations with mortality in the UK Biobank. Sci Rep 8, 12316, doi:10.1038/s41598-018-30759-1 (2018).

11 Machiela, M. J. et al. Characterization of large structural genetic mosaicism in human autosomes. Am J Hum Genet 96, 487-497, doi:10.1016/j.ajhg.2015.01.011 (2015).

12 Aw, D., Silva, A. B. \& Palmer, D. B. Immunosenescence: emerging challenges for an ageing population. Immunology 120, 435-446, doi:10.1111/j.1365-2567.2007.02555.x (2007).

13 Franceschi, C., Bonafe, M. \& Valensin, S. Human immunosenescence: the prevailing of innate immunity, the failing of clonotypic immunity, and the filling of immunological space. Vaccine 18, 1717-1720, doi:10.1016/s0264-410x(99)00513-7 (2000).

14 Ongradi, J. \& Kovesdi, V. Factors that may impact on immunosenescence: an appraisal. Immun Ageing 7, 7, doi:10.1186/1742-4933-7-7 (2010).

15 Panda, A. et al. Human innate immunosenescence: causes and consequences for immunity in old age. Trends Immunol 30, 325-333, doi:10.1016/j.it.2009.05.004 (2009).

16 Aoshi, T., Koyama, S., Kobiyama, K., Akira, S. \& Ishii, K. J. Innate and adaptive immune responses to viral infection and vaccination. Curr Opin Virol 1, 226-232, doi:10.1016/j.coviro.2011.07.002 (2011).

17 Holly, M. K., Diaz, K. \& Smith, J. G. Defensins in Viral Infection and Pathogenesis. Annu Rev Virol 4, 369-391, doi:10.1146/annurev-virology-101416-041734 (2017).

18 Pallett, L. J., Schmidt, N. \& Schurich, A. T cell metabolism in chronic viral infection. Clin Exp Immunol 197, 143-152, doi:10.1111/cei.13308 (2019).

19 Wu, P. et al. Mapping ICD-10 and ICD-10-CM Codes to Phecodes: Workflow Development and Initial Evaluation. JMIR Med Inform 7, e14325, doi:10.2196/14325 (2019). 
20 Thompson, D. J. et al. Genetic predisposition to mosaic Y chromosome loss in blood. Nature 575, 652-657, doi:10.1038/s41586-019-1765-3 (2019).

21 Consortium, G. T. The GTEx Consortium atlas of genetic regulatory effects across human tissues. Science 369, 1318-1330, doi:10.1126/science.aaz1776 (2020).

$22 \mathrm{Lu}$, Q. et al. Systematic tissue-specific functional annotation of the human genome highlights immune-related DNA elements for late-onset Alzheimer's disease. PLoS Genet 13, e1006933, doi:10.1371/journal.pgen.1006933 (2017).

23 Bick, A. G. et al. Genetic Interleukin 6 Signaling Deficiency Attenuates Cardiovascular Risk in Clonal Hematopoiesis. Circulation 141, 124-131, doi:10.1161/CIRCULATIONAHA.119.044362 (2020).

24 Genovese, G. et al. Clonal hematopoiesis and blood-cancer risk inferred from blood DNA sequence. $N$ Engl J Med 371, 2477-2487, doi:10.1056/NEJMoa1409405 (2014).

25 Jaiswal, S. et al. Age-related clonal hematopoiesis associated with adverse outcomes. $N$ Engl J Med 371, 2488-2498, doi:10.1056/NEJMoa1408617 (2014).

26 Jaiswal, S. et al. Clonal Hematopoiesis and Risk of Atherosclerotic Cardiovascular Disease. N Engl J Med 377, 111-121, doi:10.1056/NEJMoa1701719 (2017).

27 Xie, M. et al. Age-related mutations associated with clonal hematopoietic expansion and malignancies. Nat Med 20, 1472-1478, doi:10.1038/nm.3733 (2014).

28 Wang, L. et al. Integrated single-cell genetic and transcriptional analysis suggests novel drivers of chronic lymphocytic leukemia. Genome Res 27, 1300-1311, doi:10.1101/gr.217331.116 (2017).

29 de Weerdt, I. et al. Innate lymphoid cells are expanded and functionally altered in chronic lymphocytic leukemia. Haematologica 101, e461-e464, doi:10.3324/haematol.2016.144725 (2016).

30 Bartik, M. M., Welker, D. \& Kay, N. E. Impairments in immune cell function in B cell chronic lymphocytic leukemia. Semin Oncol 25, 27-33 (1998).

31 Arruga, F. et al. Immune Response Dysfunction in Chronic Lymphocytic Leukemia: Dissecting Molecular Mechanisms and Microenvironmental Conditions. Int J Mol Sci 21, doi:10.3390/ijms21051825 (2020).

32 Zhou, W. et al. Mosaic loss of chromosome $\mathrm{Y}$ is associated with common variation near TCL1A. Nat Genet 48, 563-568, doi:10.1038/ng.3545 (2016).

33 Galluzzi, L., Buque, A., Kepp, O., Zitvogel, L. \& Kroemer, G. Immunological Effects of Conventional Chemotherapy and Targeted Anticancer Agents. Cancer Cell 28, 690-714, doi:10.1016/j.ccell.2015.10.012 (2015).

34 Balkwill, F. \& Mantovani, A. Inflammation and cancer: back to Virchow? Lancet 357, 539-545, doi:10.1016/S0140-6736(00)04046-0 (2001).

35 de Visser, K. E., Eichten, A. \& Coussens, L. M. Paradoxical roles of the immune system during cancer development. Nat Rev Cancer 6, 24-37, doi:10.1038/nrc1782 (2006).

36 Lucas, C. et al. Longitudinal analyses reveal immunological misfiring in severe COVID19. Nature, doi:10.1038/s41586-020-2588-y (2020).

37 Giamarellos-Bourboulis, E. J. et al. Complex Immune Dysregulation in COVID-19 Patients with Severe Respiratory Failure. Cell Host Microbe 27, 992-1000 e1003, doi:10.1016/j.chom.2020.04.009 (2020).

38 Huang, C. et al. Clinical features of patients infected with 2019 novel coronavirus in Wuhan, China. Lancet 395, 497-506, doi:10.1016/S0140-6736(20)30183-5 (2020). 
39 Cunha, L. L., Perazzio, S. F., Azzi, J., Cravedi, P. \& Riella, L. V. Remodeling of the

2 Immune Response With Aging: Immunosenescence and Its Potential Impact on COVID-

319 Immune Response. Front Immunol 11, 1748, doi:10.3389/fimmu.2020.01748 (2020).

440 Bycroft, C. et al. The UK Biobank resource with deep phenotyping and genomic data.

$5 \quad$ Nature 562, 203-209, doi:10.1038/s41586-018-0579-z (2018).

$641 \quad$ Nagai, A. et al. Overview of the BioBank Japan Project: Study design and profile. $J$

$7 \quad$ Epidemiol 27, S2-S8, doi:10.1016/j.je.2016.12.005 (2017).

842 Smoller, J. W. et al. An eMERGE Clinical Center at Partners Personalized Medicine. $J$

$9 \quad$ Pers Med 6, doi:10.3390/jpm6010005 (2016).

1043 Peiffer, D. A. et al. High-resolution genomic profiling of chromosomal aberrations using Infinium whole-genome genotyping. Genome Res 16, 1136-1148, doi:10.1101/gr.5402306 (2006).

1344 Townsend, P., Phillimore P., Beattie A. Health and deprivation. Inequality and the North.

$14 \quad$ Health Policy 10 (1989).

1545 Finucane, H. K. et al. Partitioning heritability by functional annotation using genomewide association summary statistics. Nat Genet 47, 1228-1235, doi:10.1038/ng.3404 (2015).

$\mathrm{Hu}$, Y. et al. A statistical framework for cross-tissue transcriptome-wide association analysis. Nat Genet 51, 568-576, doi:10.1038/s41588-019-0345-7 (2019). 2016 update. Nucleic Acids Res 44, W90-97, doi:10.1093/nar/gkw377 (2016). 
a.

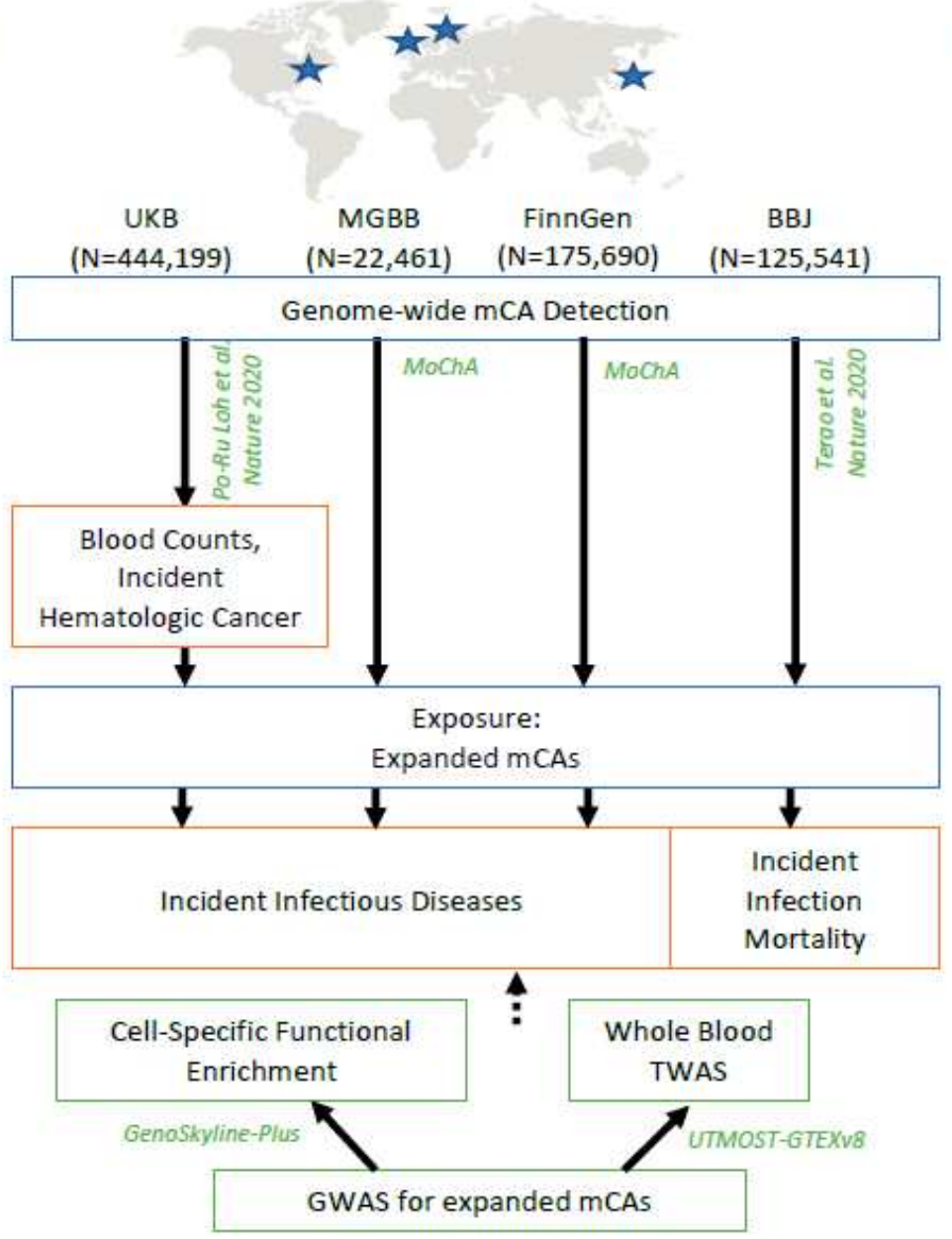

b.

All Expanded mCAs (Cell fraction $>10 \%$ )

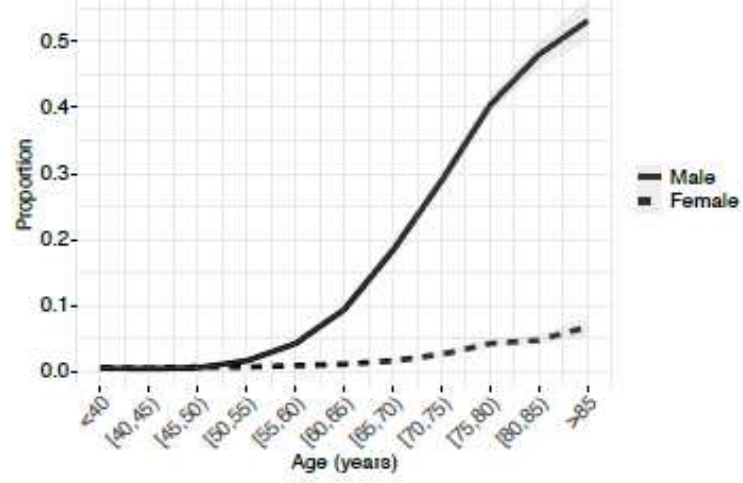

c. Expanded Autosomal mCAs (Cell fraction $>10 \%$ )

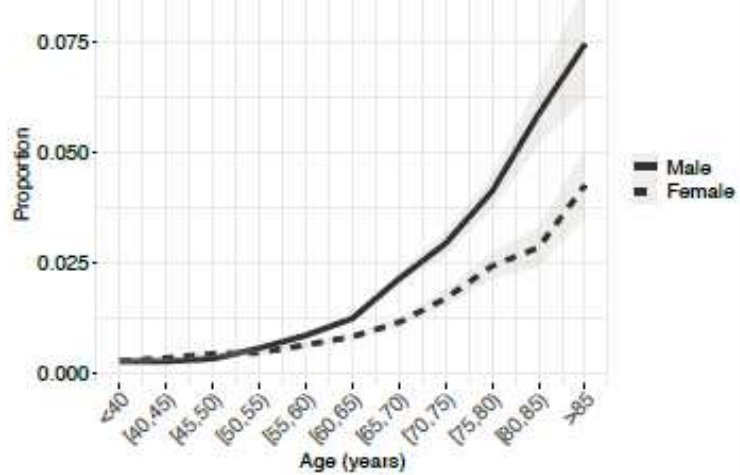

c.

All mCAs
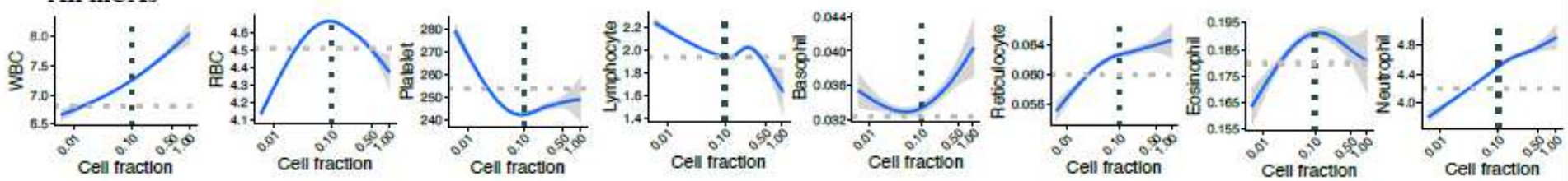

Autosomal mCAs
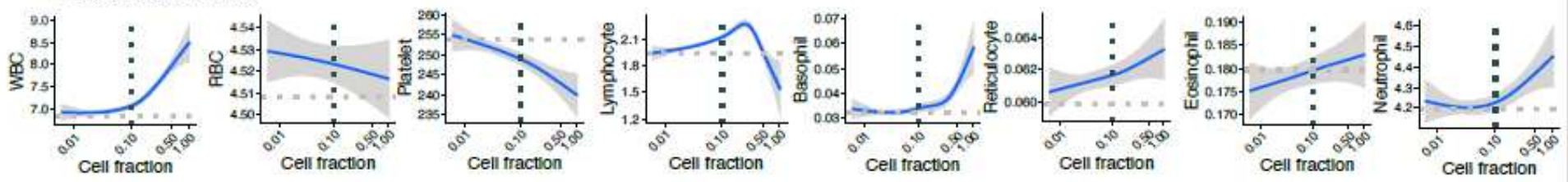

\section{Figure 1}

Study schematic. a. Genome-wide mCAs were detected across the UKB4, MGBB (via the MoChA pipeline), FinnGen (via the MoChA pipeline), and BBJ3. Association of expanded mCAs (cell fraction $>10 \%$ ) with incident infectious diseases in UKB, MGBB, and FinnGen and with incident infectious disease mortality in BBJ was performed. A GWAS for expanded mCAs was then performed in the UKB to discover causal factors for expanded mCAs. Using the GWAS results, cell-specific functional enrichment analyses were performed using GenoSkyline- Plus, which combines epigenetic and transcriptomic annotations with 
GWAS summary statistics to estimate the relative contribution of cell-specific functional markers to the GWAS results. Additionally, to prioritize putative causal genes and pathways promoting the development of expanded mCAs, whole blood TWAS was performed using UTMOST via GTEx v8. Association of $b$. all expanded mCAs with cell fraction $>10 \%$, and $c$. all expanded autosomal mCAs, with age using 5 -year age bins stratified by sex among individuals in the UKB, MGBB, FinnGen, and BBJ combined. Plots by cohort and across other mCA groupings are available in Supplementary Note 7, 8. d. Associations of mCA cell fraction with blood counts (in units of $10^{\wedge} 9$ cells $/ L$ ) in the UKB among individuals without prevalent hematologic cancer at time of blood draw for genotyping and cell count measurement. The dotted horizontal lines reflect the mean blood count for individuals without an mCA. The dotted vertical lines at cell fraction of 0.10 represents the cutoff for the expanded mCA definition. Individuals with known hematologic cancer at time of or prior to blood draw for genotyping were excluded. BBJ = BioBank Japan, GTEx v8 = Genotype-Tissue Expression project version 8, GWAS=genome-wide association study, MGBB = Mass General Brigham Biobank, $\mathrm{mCA}=$ mosaic chromosomal alterations, MoChA = Mosaic Chromosomal Alterations software (https://github.com/freeseek/mocha), TWAS = transcriptome-wide association study, UKB = UK Biobank, UTMOST = Unified Test for MOlecular SignaTures. 
a.
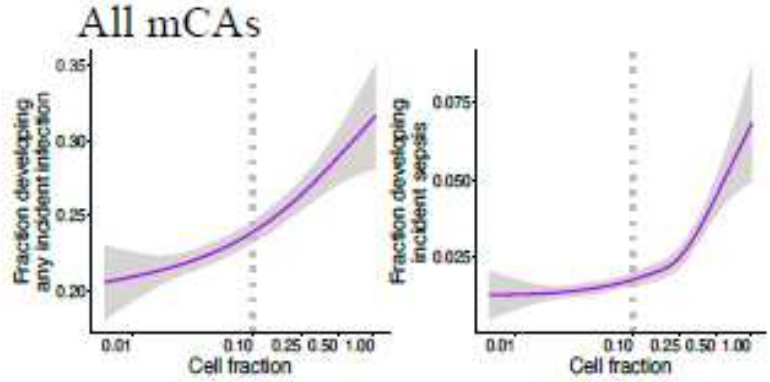

c.
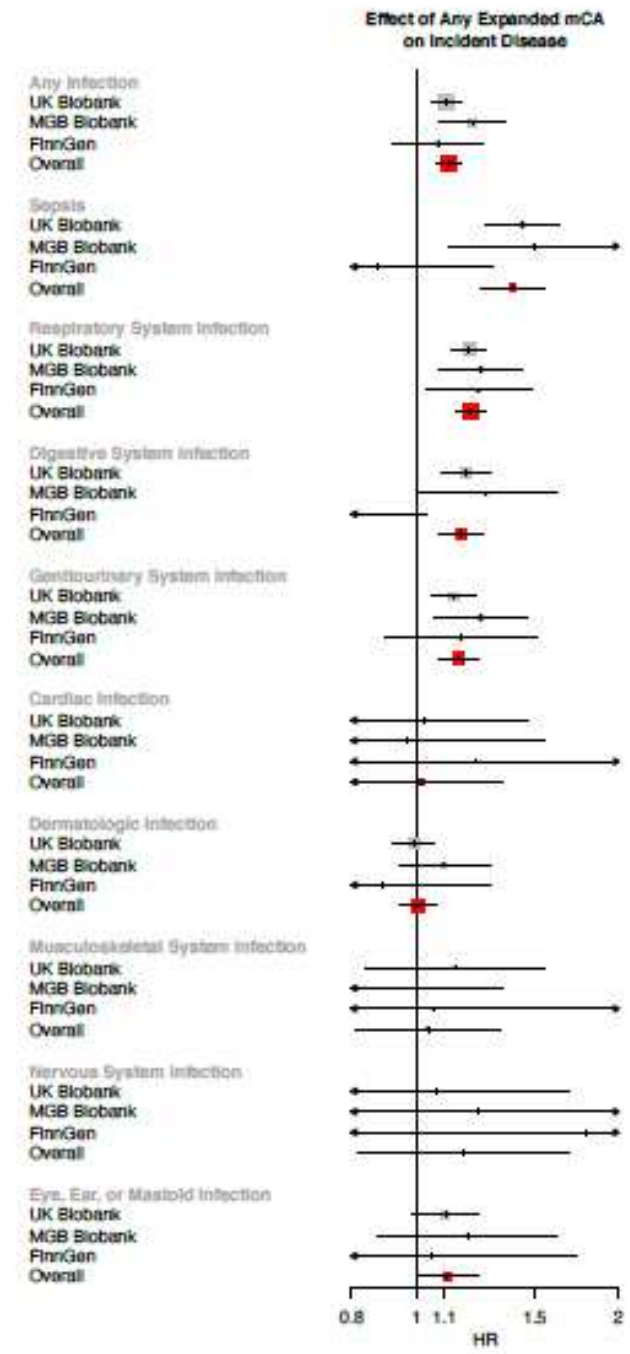

b.
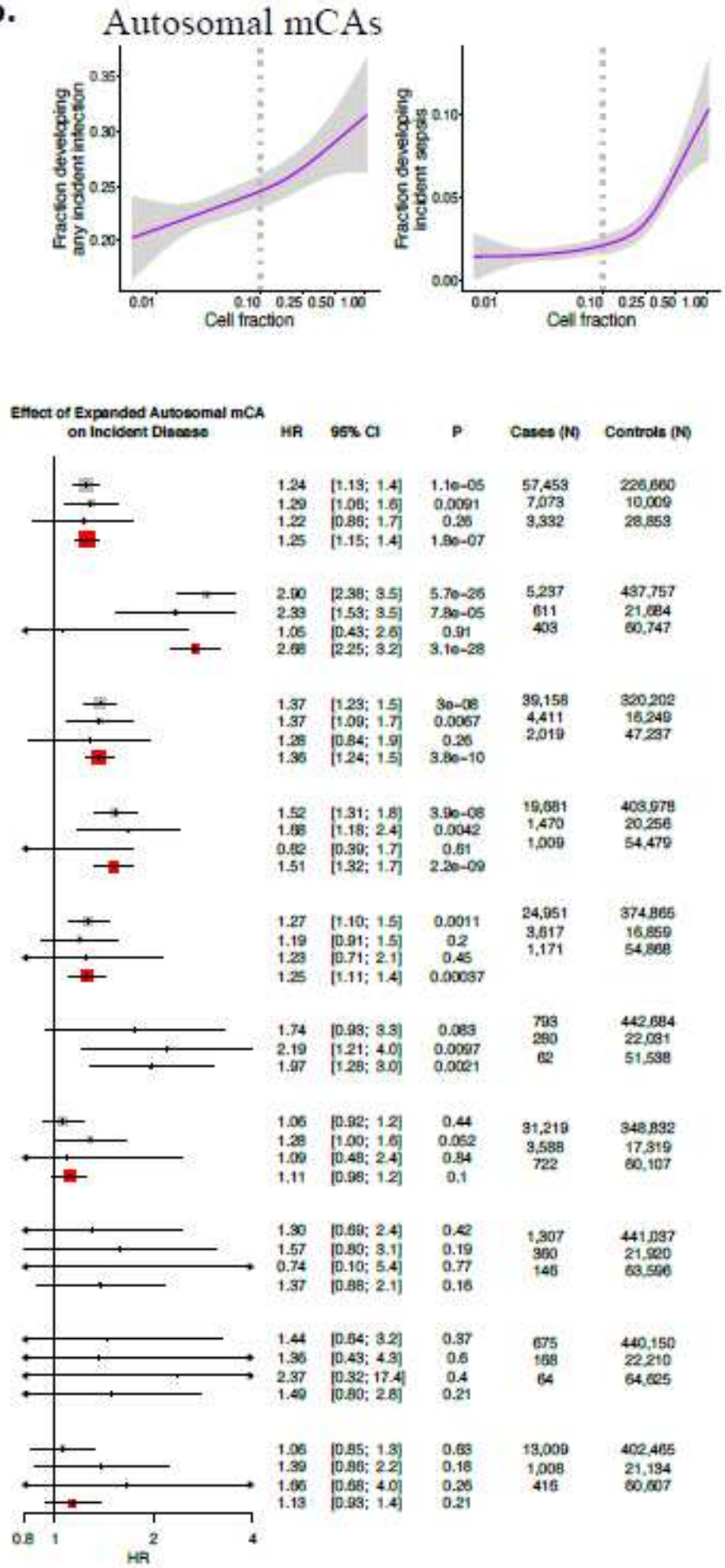

\section{Figure 2}

Associations of expanded mCAs with incident infections. Visualizing the dependence with cell fraction among a. all mCAs, and b. autosomal mCAs, of any incident infection and incident sepsis in the UKB among individuals without prevalent hematologic cancer at time of blood draw for genotyping across. The dotted vertical lines at cell fraction of 0.10 represents the cutoff for the expanded mCA definition. c. Association of all expanded mCAs, and separately, expanded autosomal mCAs with incident infections across individuals in the UKB, MGBB, and FinnGen. Analyses are adjusted for age, age2, sex, smoking status, and principal components 1-10 of ancestry. Individuals with prevalent hematologic cancer were 
excluded from analysis. Association analyses for other groupings of mCAs (including across all mCAs regardless of cell fraction, as well as chrX and chrY mCAs are provided in Supplementary Notes 10,12). BBJ = BioBank Japan, MGBB = Mass General Brigham Biobank, mCA = mosaic chromosomal alterations, UKB = UK Biobank.

a.

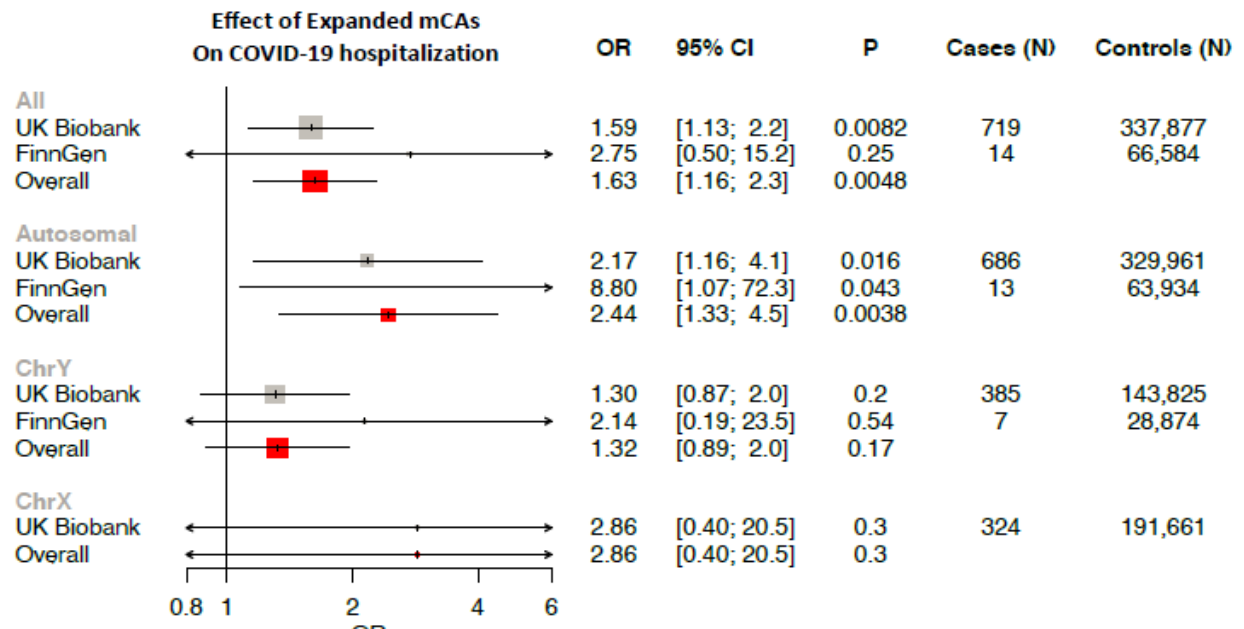

b.

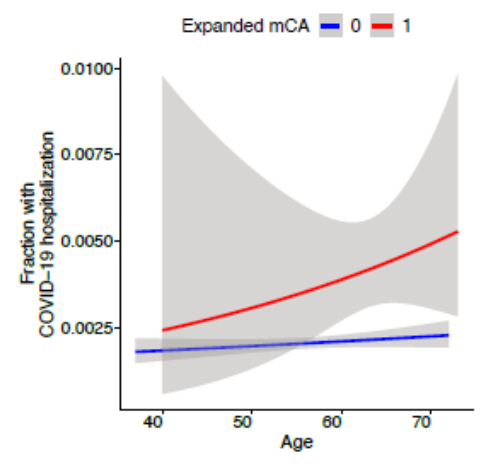

C.

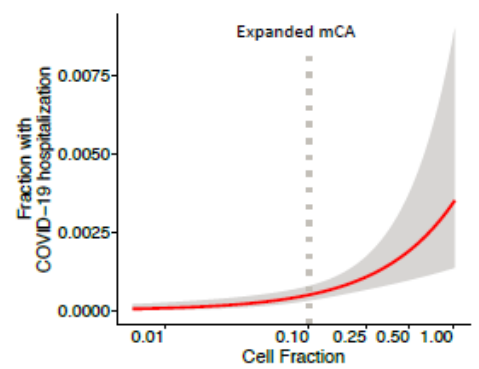

$\mathrm{OR}$
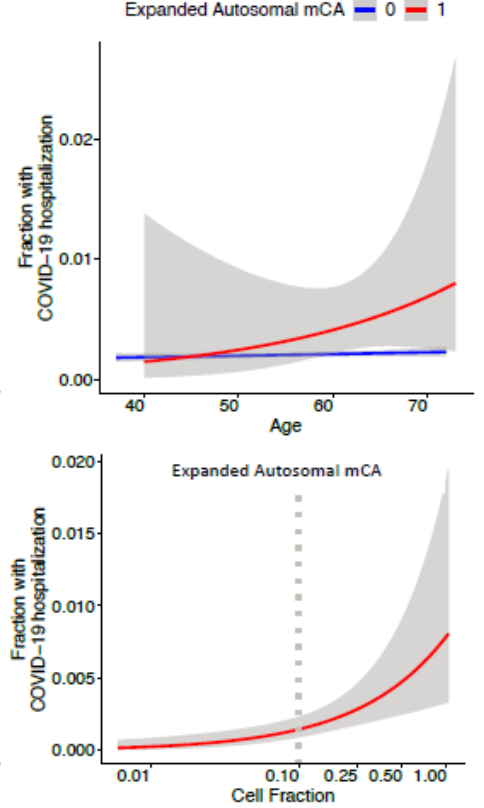

d.

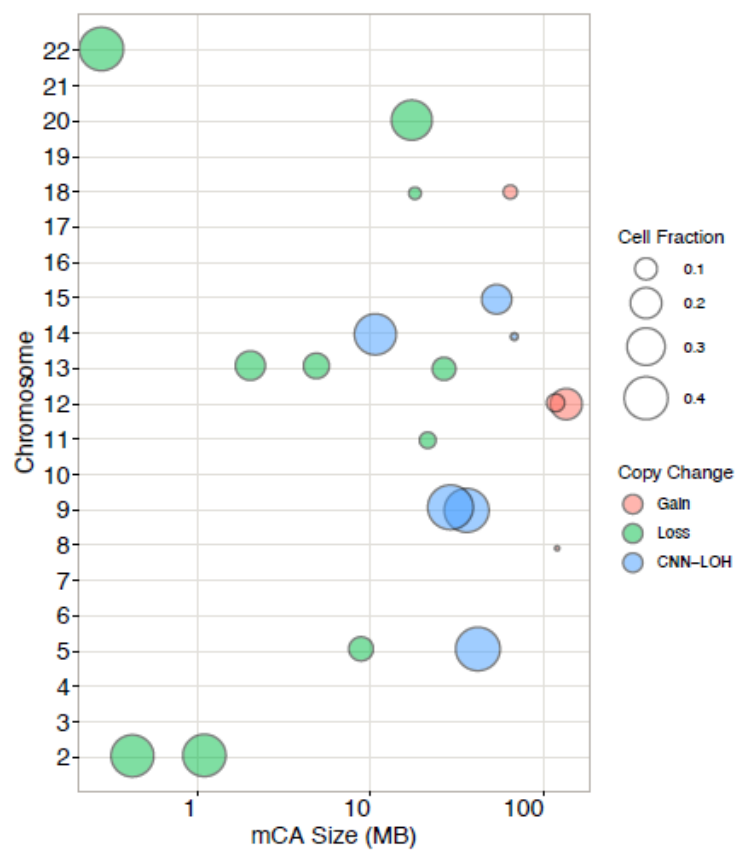

\section{Figure 3}

Association of expanded mCAs with COVID-19 Hospitalization. a. Associaiton of expanded mCAs with COVID-19 Hospitalization across the UKB and FinnGen. Individuals with known hematologic cancer at time of or prior to blood draw for genotyping were excluded. Analyses are adjusted for age, age2, sex, ever smoking status, and principal components of ancestry. b. Fraction of COVID-19 hospitalizations plotted by age, stratified by Expanded mCA (left) and expanded autosomal mCA (right) c. Fraction of COVID-19 hospitalizations plotted by cell fraction among expanded mCAs (left) and expanded autosomal mCAs (right). d.Visualization of the diverse range of expanded autosomal mCAs detected across the genome among individuals hospitalized with COVID-19 in the UK Biobank. Each point represents one mCA carried by a case, with the $x$-axis as the chromosome, $y$-axis as the mCA size in mega bases of DNA (MB). 
Additional sensitivity analyses in the UKB are provided in Extended Data Figure 8. MGBB = Mass General Brigham Biobank, UKB = UK Biobank, MB=megabase, CNN-LOH = copy number neutral loss of heterozygosity
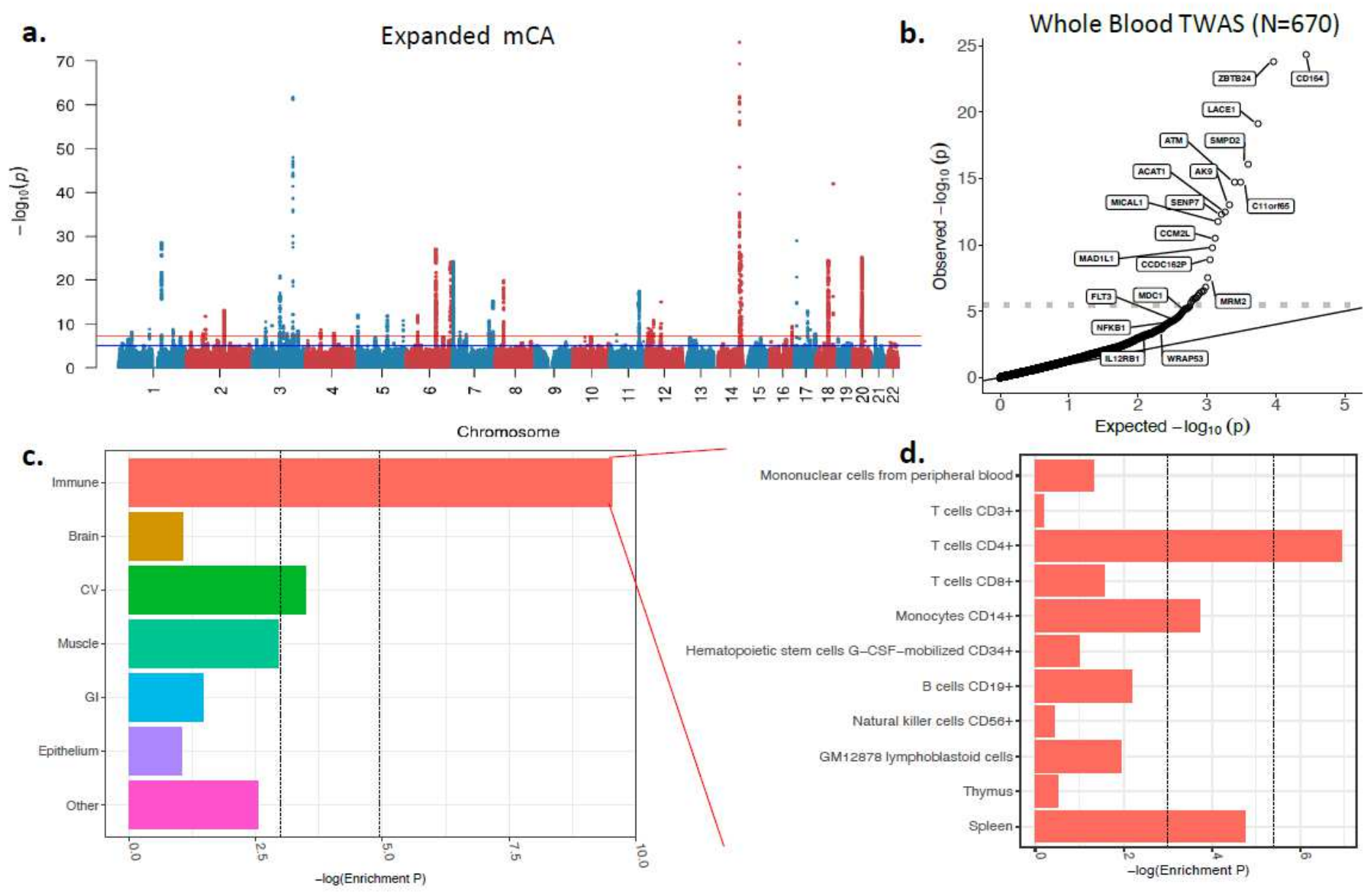

\section{Figure 4}

Inherited risk factors for expanded mCAs: GWAS, TWAS, and Cell Type Enrichment. a. GWAS for expanded mCA identified 63 independent loci. b. Quantile-quantile plot of the whole blood TWAS of the expanded mCA GWAS using 670 samples from GTExv8 shows enrichment across 62 genes. The horizontal dotted line reflects the Bonferroni-adjusted $p$-value for significance. Genes with TWAS P<5x108 or those important in the pathway-enrichment analyses from Extended Data Figure 9 are labeled. c. celltype enrichment results from the Expanded mCA GWAS across immune, brain, cardiovascular (CV), muscle, gastrointestinal (GI), epithelium, and other tissues as annotated using GenoSkyline-Plus annotations. D. Zooming in to show the stratified enrichment by specific categories of immune cells and tissues. Across panels C. and D., the vertical dotted lines indicate (1) $P=0.05$ for suggestive enrichment, and (2) the Bonferroni-adjusted P-value for significant enrichment. GWAS = genome wide association study, TWAS = transcriptome-wide association study, CV = cardiovascular, GI = Gastrointestinal

\section{Supplementary Files}


This is a list of supplementary files associated with this preprint. Click to download.

- Supplementsv4v2.pdf

- SupplementaryData18.xlsx 